\title{
Continued Fraction Algorithms for Interval Exchange Maps: an Introduction ${ }^{\star}$
}

\author{
Jean-Christophe Yoccoz
}

Collège de France, 3 Rue d'Ulm, F-75005 Paris, France

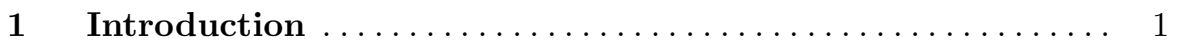

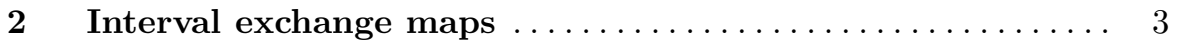

3 The Keane's property $\ldots \ldots \ldots \ldots \ldots \ldots \ldots \ldots \ldots \ldots \ldots$

4 The continuous fraction algorithm $\ldots \ldots \ldots \ldots \ldots \ldots$

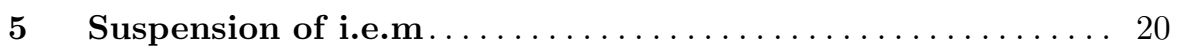

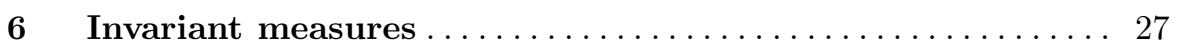

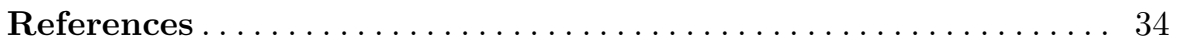

\section{Introduction}

Rotations on the circle $\mathbf{T}=\mathbf{R} / \mathbf{Z}$ are the prototype of quasiperiodic dynamics. They also constitute the starting point in the study of smooth dynamics on the circle, as attested by the concept of rotation number and the celebrated Denjoy theorem. In these two cases, it is important to distinguish the case of rational and irrational rotation number. But, if one is interested in the deeper question of the smoothness of the linearizing map, one has to solve a small divisors problem where the diophantine approximation properties of the irrational rotation number are essential. The classical continuous fraction algorithm generated by the Gauss map $G(x)=\left\{x^{-1}\right\}$ (where $x \in(0,1)$ and $\{y\}$ is the fractional part of a real number $y$ ) is the natural way to analyze or even define these approximation properties. The modular group $G L(2, \mathbf{Z})$ is here of fundamental importance, viewed as the group of isotopy classes of diffeomorphisms of $\mathbf{T}^{2}$, where act the linear flows obtained by suspension from rotations.

\footnotetext{
* to appear in "Frontiers in Number Theory, Geometry and Physics", proceedings of the Spring School at Les Houches, France, March 2003
} 
There is one obvious and classical way to generalize linear flows on the 2-dimensional torus : linear flows on higher dimensional tori. One can still define the classical diophantine approximation properties and obtain KAM-type linearization results. However, we are far from understanding these approximation properties as well as in the classical case, basically because for $n \geq 3$ the group $G L(n, \mathbf{Z})$ is far from hyperbolic and we cannot hope for a continuous fraction algorithm having all the wonderful properties it has for $n=2$.

A less obvious way to generalize linear flows on the 2-dimensional torus, but one which has received a lot of attention in recent years, is to consider linear flows on compact surfaces of higher genus called translation surfaces. We refer to Zorich's paper in this volume for a precise definition and an introduction to these very natural geometrical structures.

Linear flows on translation surfaces may be obtained as singular suspensions of one-dimensional maps of an interval called interval exchange maps (i.e.m). Such a map is obtained by cutting the interval into $d$ pieces and rearranging the pieces ; when $d=2$, this is nothing else than a rotation if the endpoints of the interval are identified to get a circle ; for $d=3$, one is still quite close to rotations (see Section 2.7 below) ; for $d \geq 4$, one can already obtain surfaces of genus $\geq 2$. Interval exchange maps (and translation surfaces) occur naturally when analyzing the dynamics of rational polygonal billiards.

An early important result is the proof by Katok-Stepin [4] that almost all i.e.m with $d=3$ are weakly mixing. Somewhat later, Keane began a systematic study of i.e.m and discovered the right concept of irrationality in this setting $([\mathrm{Ke} 1])$. He also conjectured that almost all i.e.m are uniquely ergodic. One should here beware that minimality is not sufficient to guarantee unique ergodicity, as shown by examples of Keynes-Newton [8], see also [1] and Keane [6]. Keane's conjecture was proved by Masur [11] and Veech [17] independently, see also Kerckhoff [7] and Rees [15]. The key tool developed by Veech, and also considered by Rauzy [14], is a continuous fraction algorithm for i.e.m which has most of the good properties of the classical Gauss map. However, the unique absolutely continuous invariant measure for the elementary step of this algorithm is infinite. In order to be able to apply powerful ergodic-theoretical tools such as Oseledets multiplicative ergodic theorem, one needs an absolutely continuous invariant probability measure ; this was achieved by Zorich [22] by considering an appropriate acceleration of the Rauzy-Veech continuous fraction algorithm.

Our aim in the following is to present the basic facts on the continuous fraction algorithm and its acceleration. After defining precisely interval exchange maps (Section 2), we introduce Keane's condition (Section 3), which guarantees minimality and is exactly the right condition of irrationality to start a continuous fraction algorithm. The basic step of the Rauzy-Veech algorithm is then introduced (Section 4). It appears that unique ergodicity is easily characterized in terms of the algorithm, and we give a proof of the Mazur-Veech theorem (Section 4.4). Next we explain how to suspend i.e.m to obtain linear flows on translation surfaces (Section 5). The continuous fraction algorithm 
extends to this setting and becomes basically invertible in this context. In the last chapter, we introduce Zorich's accelerated algorithm (Section 6.2) and the absolutely continuous invariant probability measure. However, we stop short of making use of this probability measure and develop the ergodic-theoretic properties of i.e.m and the continuous fraction algorithm. We refer the reader for these to [19, 20, 21, 23, 24, 25, 3].

Coming back to small divisors problems, there does not exist today a KAM-like theory of non linear perturbations of i.e.m. However, as far as the linearized conjugacy equation (also known as the cohomological equation, or the cocycle equation, or the difference equation) is concerned, Forni has obtained [2] fundamental results (in the continuous time setting) which leave some hope that such a theory could exist. Forni solves the cohomological equation (under a finite number of linear conditions) for an unspecified full measure set of i.e.m. In a jointwork with Marmi and Moussa [12], we use the continuous fraction algorithm to formulate an explicit diophantine condition (Roth type i.e.m) of full measure which allows to solve the cohomological equation (with slightly better loss of differentiability than Forni).

One last word of caution : one of the nice properties of the algorithm is its invariance under the basic time-reversal involution. However, the usual notations do not reflect this and lead by forcing an unnatural renormalization to complicated combinatorial formulas. We have thus chosen to depart from the usual notations by adopting from the start notations which are invariant under this fundamental involution. This may cause some trouble but the initial investment should be more than compensated later by simpler combinatorics.

\section{Interval exchange maps}

2.1 An interval exchange map (i.e.m) is determined by combinatorial data on one side, length data on the other side.

The combinatorial data consist of a finite set $\mathcal{A}$ of names for the intervals and of two bijections $\pi_{0}, \pi_{1}$ from $\mathcal{A}$ onto $\{1, \ldots, d\}$ (where $d=\# \mathcal{A}$ ); these indicate in which order the intervals are met between and after the map.

The length data $\left(\lambda_{\alpha}\right)_{\alpha \in \mathcal{A}}$ give the length $\lambda_{\alpha}>0$ of the corresponding interval.

More precisely, we set

$$
\begin{aligned}
& I_{\alpha}:=\left[0, \lambda_{\alpha}\right) \times\{\alpha\}, \\
& \lambda^{*}:=\sum_{\mathcal{A}} \lambda_{\alpha}, \\
& I:=\left[0, \lambda^{*}\right) .
\end{aligned}
$$

We then define, for $\varepsilon=0,1$, a bijection $j_{\varepsilon}$ from $\bigsqcup_{\mathcal{A}} I_{\alpha}$ onto $I$ :

$$
j_{\varepsilon}(x, \alpha)=x+\sum_{\pi_{\varepsilon}(\beta)<\pi_{\varepsilon}(\alpha)} \lambda_{\beta} .
$$


The i.e.m $T$ associated to these data is the bijection $T=j_{1} \circ j_{\circ}^{-1}$ of $I$.

2.2 If $\mathcal{A}, \pi_{0}, \pi_{1}, \lambda_{\alpha}$ are as above and $X: \mathcal{A}^{\prime} \rightarrow \mathcal{A}$ is a bijection, we can define a new set of data by

$$
\begin{aligned}
& \pi_{\varepsilon}^{\prime}=\pi_{\varepsilon} \circ X, \quad \varepsilon=0,1, \\
& \lambda_{\alpha^{\prime}}^{\prime}=\lambda_{X\left(\alpha^{\prime}\right)}, \alpha^{\prime} \in \mathcal{A}^{\prime} .
\end{aligned}
$$

Obviously, the "new" i.e.m $T^{\prime}$ determined by these data is the same, except for names, than the old one. In particular, we could restrict to consider normalized combinatorial data characterized by

$$
\mathcal{A}=\{1, \ldots, d\}, \pi_{0}=i d_{\mathcal{A}} .
$$

However, this leads later to more complicated formulas in the continuous fraction algorithm because the basic operations on i.e.m do not preserve normalization.

2.3 Given combinatorial data $\left(\mathcal{A}, \pi_{0}, \pi_{1}\right)$, we set, for $\alpha, \beta \in \mathcal{A}$

$$
\Omega_{\alpha, \beta}=\left\{\begin{array}{l}
+1 \text { if } \pi_{0}(\beta)>\pi_{0}(\alpha), \pi_{1}(\beta)<\pi_{1}(\alpha), \\
-1 \text { if } \pi_{0}(\beta)<\pi_{0}(\alpha), \pi_{1}(\beta)>\pi_{1}(\alpha), \\
0 \quad \text { otherwise }
\end{array}\right.
$$

The matrix $\Omega=\left(\Omega_{\alpha, \beta}\right)_{(\alpha, \beta) \in \mathcal{A}^{2}}$ is antisymmetric.

Let $\left(\lambda_{\alpha}\right)_{\alpha \in \mathcal{A}}$ be length data, and let $T$ be the associated i.e.m. For $\alpha \in$ $\mathcal{A}, y \in j_{0}\left(I_{\alpha}\right)$, we have

$$
T(y)=y+\delta_{\alpha},
$$

where the translation vector $\delta=\left(\delta_{\alpha}\right)_{\alpha \in \mathcal{A}}$ is related to the length vector $\lambda=\left(\lambda_{\alpha}\right)_{\alpha \in \mathcal{A}}$ by :

$$
\delta=\Omega \lambda
$$

2.4 There is a canonical involution $\mathcal{I}$ acting on the set of combinatorial data which exchange $\pi_{0}$ and $\pi_{1}$. For any set $\left(\lambda_{\alpha}\right)_{\alpha \in \mathcal{A}}$ of length data, the interval $I_{\alpha}, I$ are unchanged, but $j_{0}, j_{1}$ are exchanged and $T$ is replaced by $T^{-1}$. The matrix $\Omega$ is replaced by $-\Omega$ and the translation vector $\delta$ by $-\delta$.

Observe that $\mathcal{I}$ does not respect combinatorial normalization.

2.5 In the following, we will always consider only combinatorial data $\left(\mathcal{A}, \pi_{0}, \pi_{1}\right)$ which are admissible, meaning that for all $k=1,2, \ldots, d-1$, we have

$$
\pi_{0}^{-1}(\{1, \ldots, k\}) \neq \pi_{1}^{-1}(\{1, \ldots, k\})
$$


Indeed, if we had $\pi_{0}^{-1}(\{1, \ldots, k\})=\pi_{1}^{-1}(\{1, \ldots, k\})$ for some $k<d$, for any length data $\left(\lambda_{\alpha}\right)_{\alpha \in \mathcal{A}}$, the interval $I$ would decompose into two disjoint invariant subintervals and the study of the dynamics would be reduced to simpler combinatorial data.

2.6 Assume that $\# \mathcal{A}=2, \mathcal{A}=\{A, B\}$. Without loss of generality, we have $\pi_{0}(A)=\pi_{1}(B)=1, \pi_{1}(A)=\pi_{0}(B)=2$. When we identify $I=\left[0, \lambda^{*}\right)$ with the circle $\mathbf{R} / \lambda^{*} \mathbf{Z}$, the i.e.m $T$ becomes the rotation by $\lambda_{B}$.

2.7 Assume that $\# \mathcal{A}=3, \mathcal{A}=\{A, B, C\}$. Without loss of generality, we may also assume that $\pi_{0}(A)=1, \pi_{0}(B)=2, \pi_{0}(C)=3$. Amongst the 6 bijections from $\mathcal{A}$ onto $\{1,2,3\}$, there are 3 choices for $\pi_{1}$ giving rise to admissible pairs $\left(\pi_{0}, \pi_{1}\right)$, namely :

$$
\begin{aligned}
& \text { (i) } \pi_{1}(A)=2, \pi_{1}(B)=3, \pi_{1}(C)=1 ; \\
& \text { (ii) } \pi_{1}(A)=3, \pi_{1}(B)=1, \pi_{1}(C)=2 ; \\
& \text { (iii) } \pi_{1}(A)=3, \pi_{1}(B)=2, \pi_{1}(C)=1 .
\end{aligned}
$$

In case (i) and (ii), we obtain again a rotation on the circle $\mathbf{R} / \lambda^{*} \mathbf{Z}$ identified to $I$. In case (iii), consider $\hat{I}=\left[0, \lambda^{*}+\lambda_{B}\right)$ and $\hat{T}: \hat{I} \rightarrow \hat{I}$ defined by

$$
\hat{T}(y)=\left\{\begin{array}{l}
y+\lambda_{C}+\lambda_{B} \text { for } y \in\left[0, \lambda_{A}+\lambda_{B}\right) \\
y-\lambda_{A}-\lambda_{B} \text { for } y \in\left[\lambda_{A}+\lambda_{B}, \lambda^{*}+\lambda_{B}\right)
\end{array}\right.
$$

Then $\hat{T}$ is an i.e.m on $\hat{I}$. For $y \in\left[0, \lambda_{A}\right)$ or $y \in\left[\lambda_{A}+\lambda_{B}, \lambda^{*}\right)$, we have $T(y)=\hat{T}(y)$; for $y \in\left[\lambda_{A}, \lambda_{A}+\lambda_{B}\right)$, we have $\hat{T}(y) \notin I$ and $T(y)=\hat{T}^{2}(y)$. Therefore, $T$ appears as the first return map of $\hat{T}$ in $I$.

Thus, all i.e.m with $\# \mathcal{A} \leq 3$ are rotations or are closely connected to rotations.

\section{The Keane's property}

3.1 Let $T$ be an i.e.m defined by combinatorial data $\left(\mathcal{A}, \pi_{0}, \pi_{1}\right)$ and length data $\lambda=\left(\lambda_{\alpha}\right)_{\alpha \in \mathcal{A}}$. DEFinition - A connexion for $T$ is a triple $(\alpha, \beta, m)$ where $\alpha, \beta \in \mathcal{A}, \pi_{0}(\beta)>$ $1, m$ is a positive integer and $T^{m}\left(j_{0}(0, \alpha)\right)=j_{0}(0, \beta)$.

We say that $T$ has Keane's property if there is no connexion for $T$. EXERCICE 1 - For $d=2, T$ has Keane's property iff $\lambda_{A}, \lambda_{B}$ are rationally independent.

EXERCICE 2 - For $d=3$, in case (i) of 2.7 above, we have $T(y)=y+$ $\lambda_{C} \bmod \lambda^{*} \mathbf{Z}$.

Show that $T$ has Keane's property iff the two following conditions are satisfied 
1. $T$ is an irrational rotation, i.e $\lambda_{C} / \lambda^{*}$ is irrational ;

2. the points 0 and $\lambda_{A}$ are not on the same $T$-orbit, i.e there are no relations

$$
\lambda_{A}=m \lambda_{C}+n \lambda^{*}
$$

with $m, n \in \mathbf{Z}$.

3.2 THEOREM - (Keane [Ke1]) An i.e.m T with the Keane's property is minimal, i.e all orbits are dense.

Proof - Let $T$ be an i.e.m with the Keane's property.

1. We first show that $T$ has no periodic orbits. Otherwise, there exists $m>0$ s.t $P_{m}(T)=\left\{y, T^{m} y=y\right\}$ is non-empty. Then $y^{*}:=\inf P_{m}(T)$ belongs to $P_{m}(T)$. If $y^{*}>0$, there exists $k \in\{0, \ldots m-1\}$ and $\alpha \in \mathcal{A}$ such that $T^{k}\left(y^{*}\right)=j_{0}(0, \alpha)>0$ and $(\alpha, \alpha, m)$ is a connexion. If $y^{*}=0, T^{-1}\left(y^{*}\right)=$ $j_{0}(0, \alpha)>0$ for some $\alpha \in \mathcal{A}$ and $(\alpha, \alpha, m)$ is again a connexion.

2. Assume now by contradiction that there exists $y \in I$ such that $\left(T^{n}(y)\right)_{n>0}$ is not dense in $I$. Then there exists an half-open interval $J=\left[y_{0}, y_{1}\right)$ which does not contain any accumulation point of $\left(T^{n}(y)\right)_{n>0}$, nor any $j_{0}(0, \alpha)$. Let $D$ be the finite set consisting of $y_{0}, y_{1}$ and the $j_{0}(0, \alpha)$; let $D^{*}$ be the set consisting of the points $\hat{y} \in J$ such that there exists $m>0$ with $T^{m}(\hat{y}) \in D$ but $T^{l}(\hat{y}) \notin J$ for $0<l<m$. There is a canonical injective map $\hat{y} \mapsto T^{m}(\hat{y})$ from $D^{*}$ to $D$ thus $D^{*}$ is a finite set. Cut $J$ along $D^{*}$ into half open intervals $J_{1}, \ldots, J_{k}$.

For each $r \in\{1, \ldots, k\}$, there is by Poincaré recurrence a smallest $n_{r}>0$ such that $T^{n_{r}}\left(J_{r}\right) \cap J \neq \emptyset$. But then, by definition of $D^{*}$, we must have $T^{n_{r}}\left(J_{r}\right) \subset J$. We conclude that

$$
J^{*}:=\bigcup_{n \geq 0} T^{n}(J)=\bigcup_{r} \bigcup_{0 \leq n<n_{r}} T^{n}\left(J_{r}\right)
$$

is a finite union of half-open intervals, is fully invariant under $T$ (because $\left.J=\bigcup_{r} T^{n_{r}}\left(J_{r}\right)\right)$ and does not contain any accumulation point of $\left(T^{n}(y)\right)_{n \geq 0}$.

Because $\lambda^{*}$ cannot be the only accumulation point of $\left(T^{n}(y)\right)_{n \geq 0}$, we cannot have $J^{*}=I$. Because the combinatorial data are admissible (an obvious consequence of Keane's property), $J^{*}$ cannot be of the form $[0, \bar{y}), 0<\bar{y}<\lambda^{*}$.

Therefore, there exists $y^{*} \in J^{*} \cap \partial J^{*}$ with $y^{*}>0$. If $T^{l}\left(y^{*}\right) \neq j_{0}(0, \alpha)$ for all $l<0, \alpha \in \mathcal{A}$, then $T^{l}\left(y^{*}\right) \in J^{*} \cap \partial J^{*}$ for all $l \leq 0$ and $y^{*}$ is periodic. Similarly, if $T^{l}\left(y^{*}\right) \neq j_{0}(0, \alpha)$ for all $l \geq 0, \alpha \in \mathcal{A}$. Both cases are impossible by the first part of the proof. Thus there exists $l_{1}<0, l_{2} \geq 0$ and $\alpha_{1}, \alpha_{2} \in \mathcal{A}$ with $T^{l_{1}}\left(y^{*}\right)=j_{0}\left(0, \alpha_{1}\right), T^{l_{2}}\left(y^{*}\right)=j_{0}\left(0, \alpha_{2}\right)$. Taking $l_{2}$ minimal, we have $j_{0}\left(0, \alpha_{2}\right)>0$ and $\left(\alpha_{1}, \alpha_{2}, l_{2}-l_{1}\right)$ is a connexion.

\subsection{Irrationality and Keane's property}


Proposition - (Keane [Ke1]). If the length data $\left(\lambda_{\alpha}\right)_{\alpha \in \mathcal{A}}$ are rationally independent and the combinatorial data are admissible, then $T$ has Keane's property.

Proof - Assume on the opposite that there is a connexion $\left(\alpha_{0}, \alpha_{m}, m\right)$. For $0<l<m$, let $\alpha_{l} \in \mathcal{A}$ such that $T^{l}\left(j_{0}\left(0, \alpha_{0}\right)\right) \in j_{0}\left(I_{\alpha_{l}}\right)$. Denote by $\left(\delta_{\alpha}\right)_{\alpha \in \mathcal{A}}$ the translation vector. We have

$$
j_{0}\left(0, \alpha_{m}\right)-j_{0}\left(0, \alpha_{0}\right)=\sum_{0 \leq l<m} \delta_{\alpha_{l}}
$$

which, in view of 2.3 , gives

$$
\sum_{\pi_{0} \alpha<\pi_{0} \alpha_{m}} \lambda_{\alpha}-\sum_{\pi_{0} \alpha<\pi_{0} \alpha_{0}} \lambda_{\alpha}=\sum_{0 \leq l<m}\left(\sum_{\pi_{1} \alpha<\pi_{1} \alpha_{l}} \lambda_{\alpha}-\sum_{\pi_{0} \alpha<\pi_{0} \alpha_{l}} \lambda_{\alpha}\right) .
$$

Setting, for $\alpha \in \mathcal{A}$ :

$$
n_{\alpha}:=\#\left\{l \in[0, m), \pi_{1}\left(\alpha_{l}\right)>\pi_{1}(\alpha)\right\}-\#\left\{l \in(0, m], \pi_{0}\left(\alpha_{l}\right)>\pi_{0}(\alpha)\right\}
$$

we obtain $\sum n_{\alpha} \lambda_{\alpha}=0$ and therefore $n_{\alpha}=0$ for all $\alpha \in \mathcal{A}$ from rational independence.

Let $\hat{d}$ be the highest value taken by the $\pi_{1}\left(\alpha_{l}\right), l \in[0, m)$ or the $\pi_{0}\left(\alpha_{l}\right), l \in$ $(0, m]$. Because the combinatorial data are admissible, there must exists $\hat{\alpha} \in \mathcal{A}$ with $\pi_{0}(\hat{\alpha}) \geq \hat{d}$ but $\pi_{1}(\hat{\alpha})<\hat{d}$. Then $\pi_{0}\left(\alpha_{l}\right) \leq \pi_{0}(\hat{\alpha})$ for $l \in(0, m]$. As $n_{\hat{\alpha}}=0$, we must have $\pi_{1}\left(\alpha_{l}\right) \leq \pi_{1}(\hat{\alpha})<\hat{d}$ for all $l \in[0, m)$. In a symmetric way, we also prove that $\pi_{0}\left(\alpha_{l}\right)<\hat{d}$ for all $l \in(0, m]$. This contradicts the definition of $\hat{d}$.

\subsection{A continuous version of interval exchange maps}

The construction which follows is completely similar to the construction of Denjoy counter examples, i.e $C^{1}$ diffeomorphisms of the circle with no periodic orbits and a minimal invariant Cantor set.

Let $T$ be an i.e.m with combinatorial data $\left(\mathcal{A}, \pi_{0}, \pi_{1}\right)$; for simplicity we assume that $T$ has Keane's property.

For $n \geq 0$, define

$$
\begin{aligned}
& D_{0}(n)=\left\{T^{-n}\left(j_{0}(0, \alpha)\right), \alpha \in \mathcal{A}, \pi_{0}(\alpha)>1\right\}, \\
& D_{1}(n)=\left\{T^{+n}\left(j_{1}(0, \alpha)\right), \alpha \in \mathcal{A}, \pi_{1}(\alpha)>1\right\} .
\end{aligned}
$$

It follows from the Keane's property that these sets are disjoint from each other and do not contain 0 .

Define an atomic measure $\mu$ by

$$
\mu=\sum_{n \geq 0} \sum_{D_{0}(n) \sqcup D_{1}(n)} 2^{-n} \delta_{y},
$$


and then increasing maps $i^{+}, i^{-}: I \rightarrow \mathbf{R}$ by

$$
\begin{aligned}
& i^{-}(y)=y+\mu([0, y)), \\
& i^{+}(y)=y+\mu([0, y]) .
\end{aligned}
$$

We therefore have

$$
\begin{array}{ll}
i^{+}(y)<i^{-}\left(y^{\prime}\right) & \text { for } y<y^{\prime} \\
i^{+}(y)=i^{-}(y) & \text { for } y \notin \bigsqcup_{n \geq 0}\left(D_{0}(n) \sqcup D_{1}(n)\right), \\
i^{+}(y)=i^{-}(y)+2^{-n} & \text { for } y \in D_{0}(n) \sqcup D_{1}(n) .
\end{array}
$$

We also define

$$
\begin{aligned}
i^{-}\left(\lambda^{*}\right) & =\lambda^{*}+4(d-1) \\
& =\lim _{y \nearrow^{*}} i^{ \pm}(y),
\end{aligned}
$$

and

$$
\begin{aligned}
K & =\overline{i^{-}(I)} \cup i^{+}(I) \cup\left\{i^{-}\left(\lambda^{*}\right)\right\} \\
& =\overline{i^{-}(I)}=\overline{i^{+}(I)}
\end{aligned}
$$

As $T$ is minimal, $K$ is a Cantor set whose gaps are the intervals

$$
\left(i^{-}(y), i^{+}(y)\right), \quad y \in \bigcup_{n \geq 0} \bigcup_{\varepsilon} D_{\varepsilon}(n)
$$

PROPOSITION - There is a unique continuous map $\hat{T}: K \rightarrow K$ such that $\hat{T} \circ i^{+}=i^{+} \circ T$ on I. Moreover, $\hat{T}$ is a minimal homeomorphism.

Proof $-\hat{T}$ is unique because $i^{+}(I)$ is dense in $K$. Let us check that $\hat{T}$ is uniformly continuous on $i^{+}(I)$ : if $y<y^{\prime}$ satisfy $i^{+}\left(y^{\prime}\right)<i^{+}(y)+1$, it is easy to check that we have

$$
\begin{aligned}
\hat{T} \circ i^{+}\left(y^{\prime}\right)-\hat{T} \circ i^{+}(y) & =i^{+}\left(T y^{\prime}\right)-i^{+}(T y) \\
& <2\left(i^{+}\left(y^{\prime}\right)-i^{+}(y)\right) .
\end{aligned}
$$

The first statement of the proposition follows. That $\hat{T}$ is an homeomorphism follows from the observation that our setting gives symmetrical roles to $T$ and $T^{-1}$. We leave the minimality as an exercice for the reader.

\section{The continuous fraction algorithm}

\subsection{The basic operation (Rauzy [Ra], Veech [V1], [V2])}

Let $T$ be an i.e.m defined by combinatorial data $\left(\mathcal{A}, \pi_{0}, \pi_{1}\right)$ and length data $\left(\lambda_{\alpha}\right)_{\alpha \in \mathcal{A}}$. We assume as always that the combinatorial data are admissible. 
We denote by $\alpha_{0}, \alpha_{1}$ the (distinct) elements of $\mathcal{A}$ such that

$$
\pi_{0}\left(\alpha_{0}\right)=\pi_{1}\left(\alpha_{1}\right)=d
$$

Observe that if $\lambda_{\alpha_{0}}=\lambda_{\alpha_{1}}$ the triple $\left(\alpha_{1}, \alpha_{0}, 1\right)$ is a connexion and $T$ has not the Keane's property.

We now assume that $\lambda_{\alpha_{0}} \neq \lambda_{\alpha_{1}}$ and define $\varepsilon \in\{0,1\}$ by

$$
\lambda_{\alpha_{\varepsilon}}=\max \left(\lambda_{\alpha_{0}}, \lambda_{\alpha_{1}}\right)
$$

We set

$$
\begin{aligned}
& \hat{\lambda}^{*}=\lambda^{*}-\lambda_{\alpha_{1-\varepsilon}}, \\
& \hat{I}=\left[0, \hat{\lambda}^{*}\right) \subset I,
\end{aligned}
$$

and define $\hat{T}: \hat{I} \rightarrow \hat{I}$ to be the first return map of $T$ in $\hat{I}$.

When $\varepsilon=0$, we have

$$
\hat{T}(y)=\left\{\begin{array}{l}
T(y) \text { if } y \notin j_{0}\left(I_{\alpha_{1}}\right) \\
T^{2}(y) \text { if } y \in j_{0}\left(I_{\alpha_{1}}\right) .
\end{array}\right.
$$

When $\varepsilon=1$, we have similarly

$$
\hat{T}^{-1}(y)=\left\{\begin{array}{l}
T^{-1}(y) \text { if } y \notin j_{1}\left(I_{\alpha_{0}}\right), \\
T^{-2}(y) \text { if } y \in j_{1}\left(I_{\alpha_{0}}\right) .
\end{array}\right.
$$

In both case, it appears that $\hat{T}$ is again an interval exchange map which can be defined using the same alphabet $\mathcal{A}$. The length data for $\hat{T}$ are given by

$$
\left\{\begin{array}{l}
\hat{\lambda}_{\alpha}=\lambda_{\alpha} \text { if } \alpha \neq \alpha_{\varepsilon} \\
\hat{\lambda}_{\alpha_{\varepsilon}}=\lambda_{\alpha_{\varepsilon}}-\lambda_{\alpha_{1-\varepsilon}} .
\end{array}\right.
$$

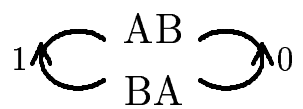

$d=2$

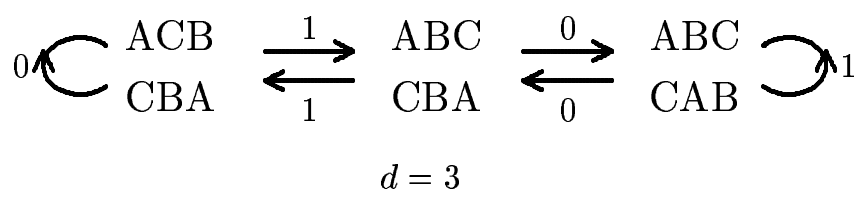

Fig. 1. Rauzy diagrams $d=2$ and $d=3$ 
The combinatorial data $\left(\hat{\pi}_{0}, \hat{\pi}_{1}\right)$ for $\hat{T}$ are given by $\hat{\pi}_{\varepsilon}=\pi_{\varepsilon}$ and

$$
\hat{\pi}_{1-\varepsilon}(\alpha)= \begin{cases}\pi_{1-\varepsilon}(\alpha) & \text { if } \pi_{1-\varepsilon}(\alpha) \leq \pi_{1-\varepsilon}\left(\alpha_{\varepsilon}\right) \\ \pi_{1-\varepsilon}(\alpha)+1 & \text { if } \pi_{1-\varepsilon}\left(\alpha_{\varepsilon}\right)<\pi_{1-\varepsilon}(\alpha)<d \\ \pi_{1-\varepsilon}\left(\alpha_{\varepsilon}\right)+1 & \text { if } \pi_{1-\varepsilon}(\alpha)=d\end{cases}
$$

We rewrite the relation between old and new length data as

$$
\lambda=V \hat{\lambda}
$$

where $V=\mathbf{1}+E_{\alpha_{\varepsilon} \alpha_{1-\varepsilon}}$ has now non negative integer coefficients an belongs to $S L\left(\mathbf{Z}^{\mathcal{A}}\right)$.

We also write

$$
\left(\hat{\pi}_{0}, \hat{\pi}_{1}\right)=R_{\varepsilon}\left(\pi_{0}, \pi_{1}\right)
$$

and observe that these new combinatorial data are admissible.

\subsection{Rauzy diagrams}

Let $\mathcal{A}$ be an alphabet. We define an oriented graph, as follows. The vertices are the admissible pairs $\left(\pi_{0}, \pi_{1}\right)$. Each vertex $\left(\pi_{0}, \pi_{1}\right)$ is the starting point of exactly two arrows with endpoints at $R_{0}\left(\pi_{0}, \pi_{1}\right)$ and $R_{1}\left(\pi_{0}, \pi_{1}\right)$. The arrow connecting $\left(\pi_{0}, \pi_{1}\right)$ to $R_{\varepsilon}\left(\pi_{0}, \pi_{1}\right)$ is said to be of type $\varepsilon$.

The operations $R_{0}, R_{1}$ are obviously invertible. Therefore each vertex is also the endpoint of exactly two arrows, one of each type.

To each arrow in the graph, we associate a name in $\mathcal{A}$ : it is the element $\alpha_{\varepsilon}$ such that $\pi_{\varepsilon}\left(\alpha_{\varepsilon}\right)=d$ (where $\left(\pi_{0}, \pi_{1}\right)$ is the starting point of the arrow and $\varepsilon$ is its type). The element $\alpha_{1-\varepsilon}$ will then be called the secondary name of this arrow.

A Rauzy diagram is a connected component in this oriented graph.
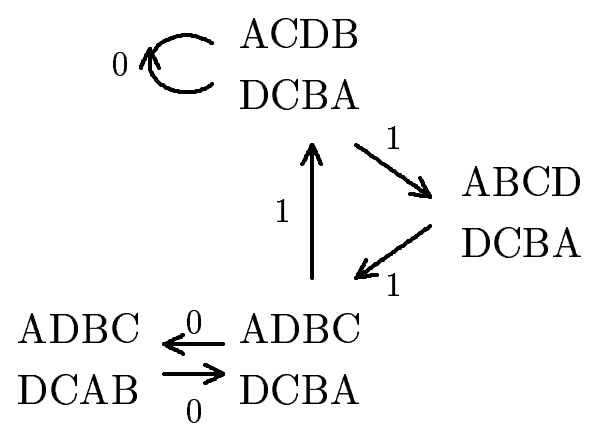
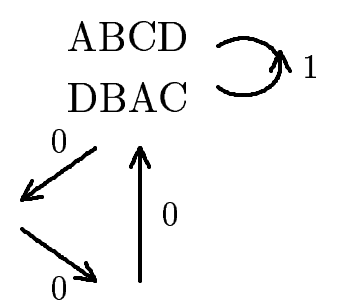

$\mathrm{ABCD} \underset{\mathrm{DACB}}{\stackrel{1}{\rightleftarrows}} \underset{1}{\stackrel{\mathrm{ABDC}}{\rightleftarrows}}$

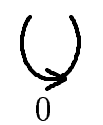

Fig. 2. Rauzy diagram $d=4$, first case 
Obviously, the Rauzy operations $R_{0}, R_{1}$ commute with change of names (cf. 2.2).

Up to change of names, there is only one Rauzy diagram with $d=\# \mathcal{A}=2$, and one with $d=\# \mathcal{A}=3$ (see figure 1 ), where the pair $\left(\pi_{0}, \pi_{1}\right)$ is denoted by the symbol

$$
\begin{aligned}
& \pi_{0}^{-1}(1) \ldots \pi_{0}^{-1}(d) \\
& \pi_{1}^{-1}(1) \ldots \pi_{1}^{-1}(d) .
\end{aligned}
$$

For $d=\# \mathcal{A}=4$, there are 2 distinct Rauzy diagrams (see figures 2 and $3)$.

In each of these diagrams, the symmetry with respect to the vertical axis corresponds to the action of the canonical involution.

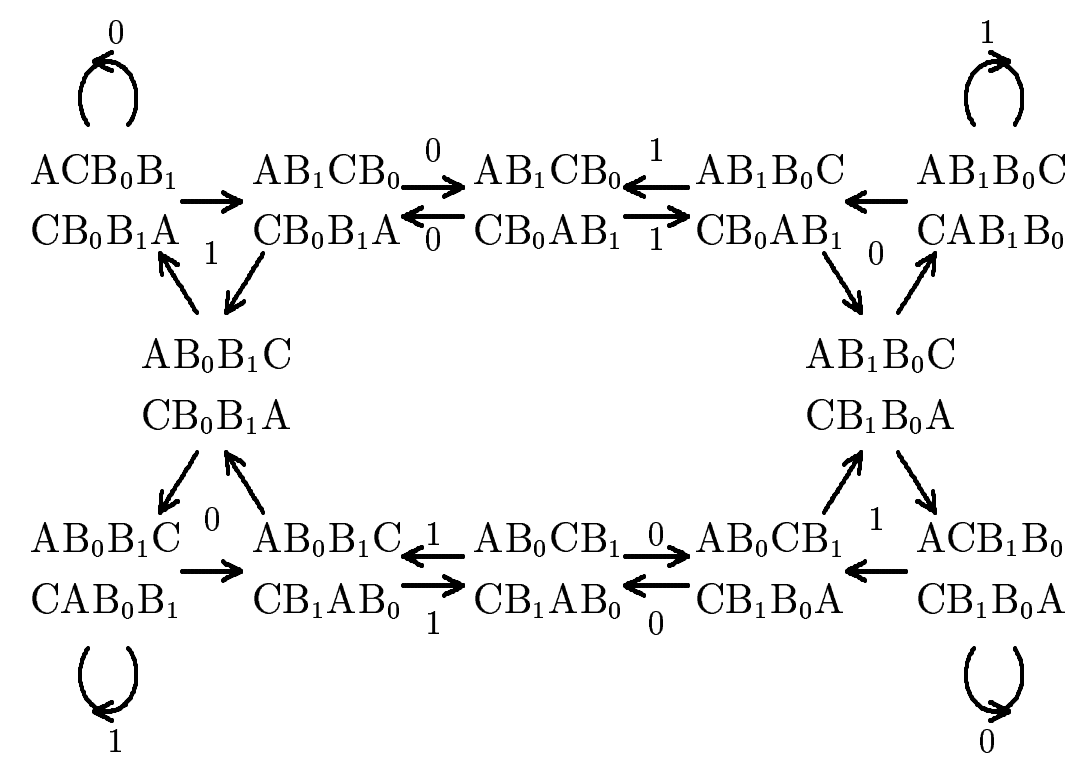

Fig. 3. Rauzy diagram $d=4$, second case

In the last diagram, there is a further symmetry with respect to the center of the diagram, which corresponds to the exchange of the names $B_{0}, B_{1}$. This is a monodromy phenomenon : to each admissible pair $\left(\pi_{0}, \pi_{1}\right)$, one can associate the permutation $\pi:=\pi_{1} \circ \pi_{0}^{-1}$ of $\{1, \ldots, d\}$, which is invariant under change of names. When we identify vertices with the same permutation, we obtain a reduced Rauzy diagram and we have a covering map from the Rauzy diagram onto the reduced Rauzy diagram.

In the first three examples above, the covering map is an isomorphism. In the last exemple, the degree of the covering map is 2 and the reduced Rauzy diagram is shown in figure 4 , where $\pi$ is denoted by $\left(\pi^{-1}(1), \ldots \pi^{-1}(d)\right)$. 


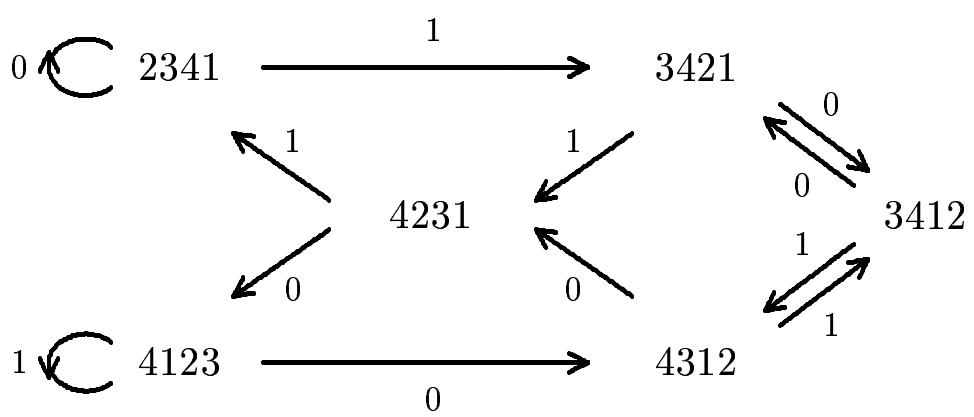

Fig. 4. Reduced Rauzy diagram $d=4$ (second case)

\subsection{Dynamics in parameter space}

Let $\mathcal{D}$ be a Rauzy diagram on an alphabet $\mathcal{A}$; denote by $V(\mathcal{D})$ the set of vertices of $\mathcal{D}$. For $\left(\pi_{0}, \pi_{1}\right) \in V(\mathcal{D})$, let

$$
\begin{aligned}
& \mathcal{C}\left(\pi_{0}, \pi_{1}\right)=\left(\mathbf{R}_{+}^{*}\right)^{\mathcal{A}} \times\left\{\left(\pi_{0}, \pi_{1}\right)\right\}, \\
& \mathcal{C}^{*}\left(\pi_{0}, \pi_{1}\right)=\left\{\left(\left(\lambda_{\alpha}\right), \pi_{0}, \pi_{1}\right) \in \mathcal{C}\left(\pi_{0}, \pi_{1}\right), \lambda_{\alpha_{0}} \neq \lambda_{\alpha_{1}}\right\}, \\
& \Delta\left(\pi_{0}, \pi_{1}\right)=\left\{\left(\left(\lambda_{\alpha}\right), \pi_{0}, \pi_{1}\right) \in \mathcal{C}\left(\pi_{0}, \pi_{1}\right), \Sigma \lambda_{\alpha}=1\right\}, \\
& \Delta^{*}\left(\pi_{0}, \pi_{1}\right)=\Delta\left(\pi_{0}, \pi_{1}\right) \cap \mathcal{C}^{*}\left(\pi_{0}, \pi_{1}\right) .
\end{aligned}
$$

For $\varepsilon \in\{0,1\}$, we also write $\Delta^{\varepsilon}\left(\pi_{0}, \pi_{1}\right), \mathcal{C}^{\varepsilon}\left(\pi_{0}, \pi_{1}\right)$ for the subsets of $\Delta^{*}\left(\pi_{0}, \pi_{1}\right), \mathcal{C}^{*}\left(\pi_{0}, \pi_{1}\right)$ defined by $\lambda_{\alpha_{\varepsilon}}>\lambda_{\alpha_{1-\varepsilon}}$.

The basic operation of 4.1 defines a 2 to 1 map from $\mathcal{C}^{*}(\mathcal{D}):=\sqcup \mathcal{C}^{*}\left(\pi_{0}, \pi_{1}\right)$ onto $\mathcal{C}(\mathcal{D}):=\sqcup \mathcal{C}\left(\pi_{0}, \pi_{1}\right)$; its restriction to $\mathcal{C}^{\varepsilon}\left(\pi_{0}, \pi_{1}\right)$ is an isomorphism onto $\mathcal{C}\left(R_{\varepsilon}\left(\pi_{0}, \pi_{1}\right)\right)$ given by the matrix $V=\mathbf{1}+E_{\alpha_{\varepsilon} \alpha_{1-\varepsilon}}$ of 4.1 . We denote this map by $\mathcal{R}$. In other terms, in the context of Section 4.1, we set

$$
\mathcal{R}(T)=\hat{T} .
$$

Because $\hat{T}$ is a first return map for $T$, if $T$ has the Keane's property, the same will be true for $\hat{T}$. This means that for such maps we will be able to iterate infinitely many times $\mathcal{R}$.

There is a canonical projection from $\mathcal{C}\left(\pi_{0}, \pi_{1}\right)$ onto $\Delta\left(\pi_{0}, \pi_{1}\right)$ which sends $\mathcal{C}^{*}\left(\pi_{0}, \pi_{1}\right)$ onto $\Delta^{*}\left(\pi_{0}, \pi_{1}\right)$. We define $\Delta(\mathcal{D})=\sqcup \Delta\left(\pi_{0}, \pi_{1}\right), \Delta^{*}(\mathcal{D})=$ $\sqcup \Delta^{*}\left(\pi_{0}, \pi_{1}\right)$, and we get a quotient map which we still denote by $\mathcal{R}$ and which is 2 to 1 from $\Delta^{*}(\mathcal{D})$ onto $\Delta(\mathcal{D})$.

Let $\left.\left(\lambda_{\alpha}\right)_{\alpha \in \mathcal{A}}, \pi_{0}, \pi_{1}\right) \in \mathcal{C}(\mathcal{D})$ be data defining an i.e.m $T$; assume that $T$ satisfies the Keane's property. Iterating $\mathcal{R}$, we get a sequence $\left(T^{(n)}\right)_{n \geq 0}$ of i.e.m with $T^{(0)}=T$. The data for $T^{(n+1)}$ are related to the data of $T^{(\bar{n})}$ by formulas :

$$
\begin{aligned}
& \left(\pi_{0}^{(n+1)}, \pi_{1}^{(n+1)}\right)=R_{\varepsilon_{n+1}}\left(\pi_{0}^{(n)}, \pi_{1}^{(n)}\right), \\
& \lambda^{(n)}=V^{(n+1)} \lambda^{(n+1)} .
\end{aligned}
$$


Denote by $\gamma^{(n+1)}$ the arrow in $\mathcal{D}$ which connects the pair $\left(\pi_{0}^{(n)}, \pi_{1}^{(n)}\right)$ to $\left(\pi_{0}^{(n+1)}, \pi_{1}^{(n+1)}\right)$. The sequence $\left(\gamma^{(n)}\right)_{n>0}$ determines an infinite path in $\mathcal{D}$ starting at $\left(\pi_{0}^{(0)}, \pi_{1}^{(0)}\right)$.

PROPOSITION - Each name in $\mathcal{A}$ is taken infinitely many times by the sequence $\left(\gamma^{(n)}\right)_{n>0}$.

Proof - Let $\mathcal{A}^{\prime}$ be the set of names which are taken infinitely many times and let $\mathcal{A}^{\prime \prime}=\mathcal{A}-\mathcal{A}^{\prime}$. Replacing $T$ by some $T^{(N)}$, we can assume that names in $\mathcal{A}^{\prime \prime}$ are not taken at all. Then the length $\lambda_{\alpha}^{(n)}, \alpha \in \mathcal{A}^{\prime \prime}$, do no depend on $n$. But then elements $\alpha \in \mathcal{A}^{\prime \prime}$ can only appear as secondary names at most finitely many times. Replacing again $T$ by some $T^{(N)}$, we can assume that secondary names are never in $\mathcal{A}^{\prime \prime}$. Then the sequences $\left(\pi_{\varepsilon}^{(n)}(\alpha)\right)_{n>0}$, for $\varepsilon \in\{0,1\}, \alpha \in \mathcal{A}^{\prime \prime}$, are non decreasing and we can assume (replacing again $T$ by $T^{(N)}$ ) that they are constant.

We now claim that we must have $\pi_{\varepsilon}^{(0)}\left(\alpha^{\prime \prime}\right)<\pi_{\varepsilon}^{(0)}\left(\alpha^{\prime}\right)$ for all $\alpha^{\prime \prime} \in \mathcal{A}^{\prime \prime}, \alpha^{\prime} \in$ $\mathcal{A}^{\prime}$ and $\varepsilon \in\{0,1\}$. Because the pair $\left(\pi_{0}^{(0)}, \pi_{1}^{(0)}\right)$ is admissible, this implies $\mathcal{A}^{\prime}=\mathcal{A}$.

To prove the claim, assume that there exist $\alpha^{\prime} \in \mathcal{A}^{\prime}, \alpha^{\prime \prime} \in \mathcal{A}^{\prime \prime}, \varepsilon \in\{0,1\}$ with $\pi_{\varepsilon}^{(0)}\left(\alpha^{\prime}\right)<\pi_{\varepsilon}^{(0)}\left(\alpha^{\prime \prime}\right)$.

As $\pi_{\varepsilon}^{(n)}\left(\alpha^{\prime \prime}\right)=\pi_{\varepsilon}^{(0)}\left(\alpha^{\prime \prime}\right)$ for all $n \geq 0$, we can never have $\pi_{\varepsilon}^{(n)}\left(\alpha^{\prime}\right)=d$ for some $n>0$. By definition of $\mathcal{A}^{\prime}$, there must exist $n \geq 0$ such that $\pi_{1-\varepsilon}^{(n)}\left(\alpha^{\prime}\right)=$ $d$; but then $\pi_{\varepsilon}^{(n+1)}\left(\alpha^{\prime \prime}\right) \neq \pi_{\varepsilon}^{(0)}\left(\alpha^{\prime \prime}\right)$, which gives a contradiction.

COROLLARY 1 - Each type and each secondary name is taken infinitely many times.

Proof - The first assertion is obvious (we do not need the proposition here). The second follows from the proposition and the following fact : if $\gamma^{(n)}, \gamma^{(n+1)}$ have not the same name, the secondary name of $\gamma^{(n+1)}$ is the (main) name of $\gamma^{(n)}$.

COROLlary 2 - The length of the intervals $I^{(n)}$ goes to 0 as $n$ goes to $\infty$.

Proof - All sequences $\left(\lambda_{\alpha}^{(n)}\right)_{n \geq 0}$ are non increasing and we want to show that they go to 0 . Let $\lambda_{\alpha}^{(\infty)}$ be the limit. Given $\varepsilon>0$, let $N \geq 0$ such that $\lambda_{\alpha}^{(N)} \leq \lambda_{\alpha}^{(\infty)}+\varepsilon$ for all $\alpha \in \mathcal{A}$. For each $\alpha \in \mathcal{A}$, there exists $n>N$ such that $\alpha$ is a secondary name for $\gamma^{(n)}$; this implies that $\lambda_{\alpha}^{(\infty)} \leq \lambda_{\alpha}^{(n)} \leq \varepsilon$ and concludes the proof.

COROLLARY 3 - Let $T$ be an i.e.m with admissible combinatorial data which does not have the Keane's property. Then the continuous fraction algorithm stops because at some point the equality $\lambda_{\alpha_{0}}^{(n)}=\lambda_{\alpha_{1}}^{(n)}\left(\right.$ with $\pi_{0}^{(n)}\left(\alpha_{0}\right)=\pi_{1}^{(n)}\left(\alpha_{1}\right)=d$ ) holds.

Proof - Let $(\alpha, \beta, m)$ a connexion for $T=T^{(0)}$. We show by infinite descent that the algorithm has to stop. Set $y_{0}=j_{0}((0, \beta))$; set $y_{1}=j_{1}((0, \alpha))$ if $\pi_{1}(\alpha) \neq 1, y_{1}=T(0)$ if $\pi_{1}(\alpha)=1$. We have $T^{\bar{m}}\left(y_{1}\right)=y_{0}$ with $\bar{m}=m-1$ if $\pi_{1}(\alpha) \neq 1, \bar{m}=m-2$ if $\pi_{1}(\alpha)=1$, and $\bar{m} \geq 0$ in both cases, with $y_{0}, y_{1}>0$. 
Assume by contradiction that the algorithm never stops. Observe that the proposition and the corollaries 1 and 2 hold, because the Keane's property was not used in their proof. Let $n$ be the largest integer such that

$$
\left|I^{(n)}\right|>\max \left(y_{0}, y_{1}\right),
$$

where $I^{(n)}$ is the domain for $T^{(n)}$. Such an $n$ exists by Corollary 2. If we had $y_{0}=y_{1}$, the equality case would happen at the next step of the basic operation. We therefore have $\bar{m}>0, y_{0} \neq y_{1}$. Assume for instance that $y_{1}>y_{0}$ (the other case is symmetric). First, because $T^{(n)}$ is the first return map of $T$ into $I^{(n)}$, there exists $0<\hat{m} \leq \bar{m}$ such that $\left(T^{(n)}\right)^{\hat{m}}\left(y_{1}\right)=y_{0}$. Second, by the definition of the basic operation, $y_{1}^{\prime}=T^{(n)}\left(y_{1}\right)$ is equal to $j_{1}\left(0, \alpha_{1}\right)$ at step $n+1$, where $\pi_{1}^{(n)}\left(\alpha_{1}\right)=d$. Then $\left(T^{(n)}\right)^{\hat{m}-1}\left(y_{1}^{\prime}\right)=y_{0}$ and therefore $\left(\right.$ as $T^{(n+1)}$ is a first return map of $\left.T^{(n)}\right)$ there exists $\bar{m}^{\prime} \leq \hat{m}-1<\bar{m}$ such that $T^{(n+1)}\left(y_{1}^{\prime}\right)=y_{0}$. We have completed one step of the descent argument, and this concludes the proof.

COROLLARY 4 - For each $m \geq 0$, there exists $n>m$ such that the matrix $Q:=V^{(m+1)} \ldots V^{(n)}$ satisfies $Q_{\alpha \beta}>0$ for all $\alpha, \beta \in \mathcal{A}$.

Proof - Write $Q=Q(n)$. Let $\alpha, \beta \in \mathcal{A}$; if $Q_{\alpha \beta}\left(n_{0}\right)>0$ for some $n_{0}$, then $Q_{\alpha \beta}(n)>0$ for all $n \geq n_{0}$ : indeed the diagonal terms of the $V$ matrices are equal to 1 . It therefore suffices to prove that for all $\alpha, \beta \in \mathcal{A}$ there exists $n_{0}$ such that $Q_{\alpha \beta}\left(n_{0}\right)>0$. Fix $\alpha, \beta \in \mathcal{A}$. If $\alpha=\beta$, we already have $Q_{\alpha \beta}(m+1)=$ 1. Assume $\alpha \neq \beta$. Let $n_{1}>m$ the smallest integer such that the arrow $\gamma^{\left(n_{1}\right)}$ has name $\alpha$. Set $\alpha_{1}:=\alpha$ and let $\alpha_{2}$ be the secondary name of $\gamma^{\left(n_{1}\right)}$; we have $Q_{\alpha_{1} \alpha_{i}}\left(n_{1}\right)>0$ for $i=1,2$. If $\beta=\alpha_{2}$, we are done. Otherwise, $d \geq 3$ and there exists a smallest integer $n_{1}^{\prime}>n_{1}$ such that the name of $\gamma^{\left(n_{1}^{\prime}\right)}$ is not $\alpha_{1}$ or $\alpha_{2}$. There also exists a smallest integer $n_{2}>n_{1}^{\prime}$ such that the name of $\gamma^{\left(n_{2}\right)}$ is $\alpha_{1}$ or $\alpha_{2}$. Then, the secondary name $\alpha_{3}$ of $\gamma^{\left(n_{2}\right)}$ is the name of $\gamma^{\left(n_{2}-1\right)}$ and therefore is different from $\alpha_{1}$ or $\alpha_{2}$. We have $V_{\alpha_{j} \alpha_{3}}^{\left(n_{2}\right)}=1$ for some $j \in\{1,2\}$, and therefore $Q_{\alpha_{1} \alpha_{i}}\left(n_{2}\right)>0$ for $i \in\{1,2,3\}$. If $\beta=\alpha_{3}$ we are done. Otherwise $d \geq 4$ and we define $n_{2}^{\prime}>n_{2}, n_{3}>n_{2}^{\prime}, \alpha_{4} \notin\left\{\alpha_{1}, \alpha_{2}, \alpha_{3}\right\}$ as above ... At some point we must have $\beta=\alpha_{j}$.

COROLlaRY 5-Define a decreasing sequence of open simplicial cones in $\mathbf{R}^{\mathcal{A}}$ by

$$
\mathcal{C}^{(0)}=\left(\mathbf{R}_{+}^{*}\right)^{\mathcal{A}}, \mathcal{C}^{(n+1)}=V^{(n+1)} \mathcal{C}^{(n)}
$$

and let $\mathcal{C}^{(\infty)}=\cap \mathcal{C}^{(n)}$. Then $\mathcal{C}^{(\infty)} \cup\{0\}$ is a closed simplicial cone, of dimension $<d=\# \mathcal{A}$.

Proof - From Corollary 4 it follows that for all $m \geq 0$ there exists $n>m$ such that the closure of $\mathcal{C}^{(n)}$ is contained in $\mathcal{C}^{(m)} \cup\{0\}$. This shows that $\mathcal{C}^{(\infty)} \cup\{0\}$ is closed. For $n \geq 0, \alpha \in \mathcal{A}$, let $e_{\alpha}^{(n)}=V^{(1)} \ldots V^{(n)}\left(e_{\alpha}\right)$, where $\left(e_{\alpha}\right)_{\alpha \in \mathcal{A}}$ is the canonical base of $\mathbf{R}^{\mathcal{A}}$. Let $n_{k}$ be an increasing sequence of integers such that $e_{\alpha}^{\left(n_{k}\right)}\left\|e_{\alpha}^{\left(n_{k}\right)}\right\|^{-1}$ converge towards a limit $e_{\alpha}^{(\infty)}$ for every $\alpha \in \mathcal{A}$. Then we must have 


$$
\mathcal{C}^{(\infty)} \cup\{0\}=\left\{\sum_{\mathcal{A}} t_{\alpha} e_{\alpha}^{(\infty)}, t_{\alpha} \geq 0\right\}
$$

The limits $e_{\alpha}^{(\infty)}$ cannot be all distinct, because all coefficients of $V^{(1)} \ldots V^{(n)}$ go to $\infty$ as $n$ goes to $\infty$ (by Corollary 4 ), and these matrices are unimodular. Thus $\mathcal{C}^{(\infty)} \cup\{0\}$ is closed, polyhedral of dimension $<d$. Indeed it is simplicial because, as we will see in the next section, it can be interpreted as a cone of invariant measures.

\subsection{Unique ergodicity and the continuous fraction algorithm}

Recall that a transformation is uniquely ergodic if it has exactly one invariant probability measure.

For an i.e.m $T$, (normalized) Lebesgue measure is invariant, hence there should be no other invariant probability measure.

Let $T$ be an i.e.m with the Keane's property. In particular, $T$ is minimal. Therefore, every finite invariant measure $\mu$ is continuous and supported by the whole of $I$. For such a measure, we set

$$
H_{\mu}(x)=\mu([0, x)) .
$$

This defines an homeomorphism from $I$ onto $I_{\mu}:=[0, \mu(I))$. Let

$$
T_{\mu}=H_{\mu} \circ T \circ H_{\mu}^{-1} .
$$

This is a one-to-one transformation of $I_{\mu}$. Actually, $T_{\mu}$ is immediately seen to be an i.e.m on $I_{\mu}$, whose combinatorial data are the same as for $T$, and whose length data $\left(\lambda_{\alpha}(\mu)\right)_{\alpha \in \mathcal{A}}$ are given by

$$
\lambda_{\alpha}(\mu)=\mu\left(j_{0}\left(I_{\alpha}\right)\right)=\mu\left(j_{1}\left(I_{\alpha}\right)\right) .
$$

Obviously, the image of $\mu$ under the conjugacy $H_{\mu}$ is the Lebesgue measure on $I_{\mu}$.

PROPOSITION - The map $\mu \mapsto\left(\lambda_{\alpha}(\mu)\right)_{\alpha \in \mathcal{A}}$ is a linear homeomorphism from the set of T-invariant finite measures onto the cone $\mathcal{C}^{(\infty)}$ of Corollary 5 In particular, $T$ is uniquely ergodic if and only if $\mathcal{C}^{(\infty)}$ is a ray.

Proof - The map is obviously linear and continuous ; as $T$ and $T_{\mu}$ are topologically conjugated, $T_{\mu}$ has also the Keane's property ; moreover, the restriction of $H_{\mu}$ to $I^{(n)}$ is an homeomorphism on $I_{\mu}^{(n)}$ which conjugates $T^{(n)}$ and $T_{\mu}^{(n)}$. Thus, the length vector $\left(\lambda_{\gamma}(\mu)\right)_{\alpha \in \mathcal{A}}$ belongs to $\mathcal{C}^{(n)}$ for every $n \geq 0$ and therefore to $\mathcal{C}^{(\infty)}$. Conversely, let $\left(\tilde{\lambda}_{\alpha}\right)_{\alpha \in \mathcal{A}}$ be a length vector in $\mathcal{C}^{(\infty)}$. Let $\tilde{T}$ be the i.e.m defined by this length vector and the same combinatorial data than $T$. The continuous fraction algorithm for $\tilde{T}$ never stops (with the same path in the Rauzy diagram than for $T$ ), hence $\tilde{T}$ has the Keane's property ; the same is true for the i.e.m. $\tilde{T}_{t}$ whose length vector is $(1-t) \lambda+t \tilde{\lambda} \in \mathcal{C}^{(\infty)}$. Therefore, 
for each $t \in[0,1]$, the points $\left(T_{t}^{k}(0)\right)_{k \geq 0}$ are distinct, form a dense set in $I_{t}$ and we have

$$
T_{t}^{k}(0)>T_{t}^{l}(0) \Longleftrightarrow T_{t^{\prime}}^{k}(0)>T_{t^{\prime}}^{l}(0)
$$

for all $k, l \geq 0, t, t^{\prime} \in[0,1]$. If we set

$$
H\left(T^{k}(0)\right)=\tilde{T}^{k}(0),
$$

for all $k \geq 0$, the map $H$ extends in a unique way to an homeomorphism from $I$ onto $\tilde{I}$ which conjugates $T$ and $\tilde{T}$. If $\mu$ is the image of Lebesgue measure under $H^{-1}$, then $\mu$ is a finite $T$-invariant measure on $I$ and $\tilde{T}=T_{\mu}$.

For $d \leq 3$, interval exchange maps are rotations or first return maps of rotations and thus are uniquely ergodic if minimal. On the other hand, Keane has constructed ([Ke2], see also [KN], [Co] $)$ i.e.m with $d=4$ which are minimal but not uniquely ergodic. Nevertheless, we have the following fundamental result :

THEOREM - (Mazur [Ma], Veech [V2]) Let $\left(Q, \pi_{0}, \pi_{1}\right)$ be any admissible combinatorial data. Then, for almost all length data $\left(\lambda_{\alpha}\right)_{\alpha \in \mathcal{A}}$, the associated i.e.m is (minimal and) uniquely ergodic.

Proof - We will give a slightly simplified version of the proof of Kerchkoff $([\operatorname{Ker}])$. Let $\mathcal{D}$ be the Rauzy diagram which contains the combinatorial data $\left(\mathcal{A}, \pi_{0}, \pi_{1}\right)$.

For any finite path $\gamma=\left(\gamma^{(i)}\right)_{0<i \leq n}$ in $\mathcal{D}$ starting at $\left(\pi_{0}, \pi_{1}\right)$, let $\left(V^{(i)}\right)_{0<i \leq n}$ be the associated matrices; let

$$
\begin{aligned}
Q(\gamma) & =V^{(1)} \ldots V^{(n)} \\
\mathcal{C}(\gamma) & =Q(\gamma)\left[\left(\mathbf{R}_{+}^{*}\right)^{\mathcal{A}}\right] \times\left\{\left(\pi_{0}, \pi_{1}\right)\right\} \\
\Delta(\gamma) & =\mathcal{C}(\gamma) \cap \Delta\left(\pi_{0}, \pi_{1}\right)
\end{aligned}
$$

For $\beta \in \mathcal{A}$, we also write

$$
Q_{\beta}(\gamma)=\sum_{\alpha} Q_{\alpha \beta}(\gamma)
$$

LEMMA 1 - We have

$$
\operatorname{vol}_{d-1}(\Delta(\gamma))=\left(\prod_{\beta} Q_{\beta}(\gamma)\right)^{-1} \operatorname{vol}_{d-1}\left(\Delta\left(\pi_{0}, \pi_{1}\right)\right) .
$$

Proof - Indeed, $Q(\gamma)$ is unimodular and we have, for $\lambda^{(0)}=Q(\gamma) \lambda^{(n)}$ :

$$
\sum_{\alpha} \lambda_{\alpha}^{(0)}=\sum_{\beta} Q_{\beta}(\gamma) \lambda_{\beta}^{(n)}
$$

LEMMA 2 - Let $C \geq 1$ a constant such that

$$
\max _{\alpha} Q_{\alpha}(\gamma) \leq C \min _{\alpha} Q_{\alpha}(\gamma)
$$


There exists a constant $c \in(0,1)$, depending only on $C$ and $d$, and a path $\gamma^{\prime}$ extending $\gamma$ such that

$$
\begin{aligned}
& \operatorname{vol}_{d-1}\left(\Delta\left(\gamma^{\prime}\right)\right) \geq c \operatorname{vol}_{d-1}(\Delta(\gamma)), \\
& \operatorname{diam}\left(\Delta\left(\gamma^{\prime}\right)\right) \leq(1-c) \operatorname{diam}(\Delta(\gamma)) .
\end{aligned}
$$

Proof - Choose a path $\tilde{\gamma}$ starting from the endpoint of $\gamma$ such that $Q_{\alpha \beta}(\tilde{\gamma})>0$ for all $\alpha, \beta \in \mathcal{A}$. We have $Q_{\alpha \beta}(\tilde{\gamma}) \leq C_{1}$, with $C_{1}$ depending only on $d$. Let $\gamma^{\prime}=\gamma \star \tilde{\gamma}$. We have, for $\beta \in \mathcal{A}$

$$
Q_{\beta}\left(\gamma^{\prime}\right)=\sum_{\alpha} Q_{\alpha}(\gamma) Q_{\alpha \beta}(\tilde{\gamma})
$$

and thus, by Lemma 1

$$
\operatorname{vol}_{d-1}\left(\Delta\left(\gamma^{\prime}\right)\right) \geq\left(C C_{1} d\right)^{-d} \operatorname{vol}_{d-1}(\Delta(\gamma)) .
$$

It is also clear, considering orthogonal projections on 1-dimensional lines, that we have

$$
\operatorname{diam}\left(\Delta\left(\gamma^{\prime}\right)\right) \leq\left(1-\frac{2}{C_{1}(d-1)+1}\right) \operatorname{diam}(\Delta(\gamma)) .
$$

LEMMA 3 - Let $\left(\pi_{0}^{(n)}, \pi_{1}^{(n)}\right)$ be the vertex of $\mathcal{D}$ endpoint of $\gamma$; define $\alpha_{0}, \alpha_{1} \in \mathcal{A}$ by $\pi_{\varepsilon}^{(n)}\left(\alpha_{\varepsilon}\right)=d, \varepsilon=0,1$. For $\varepsilon=0,1$, let $\Delta^{\varepsilon}(\gamma)$ be formed of those length data in $\Delta(\gamma)$ for which the $(n+1)^{\text {th }}$ arrow has type $\varepsilon$. Then

$$
\operatorname{vol}_{d-1}\left(\Delta^{\varepsilon}(\gamma)\right)=\frac{Q_{\alpha_{1-\varepsilon}}(\gamma)}{Q_{\alpha_{0}}(\gamma)+Q_{\alpha_{1}}(\gamma)} \operatorname{vol}_{d-1}(\Delta(\gamma)) .
$$

Proof - Clear from Lemma 1.

Let $T$ be an i.e.m in $\Delta(\gamma)$ satisfying Keane's condition, and let $\left(\gamma^{(i)}(T)\right)_{i \geq 0}$ be the associated path ; we therefore have $\gamma^{(i)}(T)=\gamma^{(i)}$ for $0<i \leq n$. Let $\left(V^{(i)}(T)\right)_{i \geq O}$ be the associated matrices ; define

$$
Q(i, T)=V^{(1)}(T) \ldots V^{(i)}(T) .
$$

Fix $\alpha \in \mathcal{A}$, and define $Q_{\alpha}^{\prime}(T)=Q_{\alpha}(n(\alpha, T), T)$, where $n(\alpha, T)$ is the smallest integer $m>n$ such that the name of $\gamma^{(m)}(T)$ is $\alpha$ (this is well defined by the proposition in 4.3). We then have :

LEMMA 4 - For any $\mathcal{C} \geq 1$, we have :

$$
\operatorname{vol}_{d-1}\left(\left\{T \in \Delta(\gamma), Q_{\alpha}^{\prime}(T) \geq \mathcal{C} Q_{\alpha}(\gamma)\right\}\right) \leq \mathcal{C}^{-1} \operatorname{vol}_{d-1}(\Delta(\gamma)) .
$$

Proof - We will show the slightly stronger result that the inequality of the lemma holds even after conditioning by the value $\bar{n}$ of $n(\alpha, T)-n$. We show this last result by induction on $\bar{n}$. 
We have $\bar{n}=1$ iff the name of $\gamma^{(n+1)}(T)$ is $\alpha$; in this case, we have $Q_{\alpha}^{\prime}(T)=Q_{\alpha}(\gamma)$ and the estimate holds for all $\mathcal{C} \geq 1$.

If $\pi_{0}^{(n)}(\alpha)<d$ and $\pi_{1}^{(n)}(\alpha)<d$, we divide $\Delta(\gamma)$ into $\Delta^{0}(\gamma)$ and $\Delta^{1}(\gamma)$ and apply the induction hypothesis to both simplices to conclude.

Assume on the other hand that $\pi_{0}^{(n)}(\alpha)<d, \pi_{1}^{(n)}(\alpha)=d$; if $\bar{n}>1$, the name of $\gamma^{(n+1)}(T)$ is the element $\alpha_{0} \in \mathcal{A}$ such that $\pi_{0}^{(n)}\left(\alpha_{0}\right)=d$ and we have

$$
\begin{aligned}
& Q_{\alpha}(n+1, T)=Q_{\alpha}(\gamma)+Q_{\alpha_{0}}(\gamma), \\
& \operatorname{vol}_{d-1}\left(\Delta^{0}(\gamma)\right)=\frac{Q_{\alpha}(\gamma)}{Q_{\alpha}(n+1, T)} \operatorname{vol}_{d-1}(\Delta(\gamma)),
\end{aligned}
$$

by Lemma 3 . We will have $Q_{\alpha}^{\prime}(T) \geq Q_{\alpha}(n+1, T)$. If $1 \leq \mathcal{C} \leq\left(Q_{\alpha}(\gamma)\right)^{-1} Q_{\alpha}(n+$ $1, T)$, the estimate of the lemma holds immediately. For $\mathcal{C}>\left(Q_{\alpha}(\gamma)\right)^{-1} Q_{\alpha}(n+$ $1, T)$, we set

$$
\begin{aligned}
& \mathcal{C}^{\prime}=\mathcal{C} Q_{\alpha}(\gamma)\left(Q_{\alpha}(n+1, T)\right)^{-1}, \\
& \gamma^{\prime}=\gamma \star \gamma^{(n+1)}(T)
\end{aligned}
$$

and use the induction hypothesis to conclude. The case $\pi_{0}^{(n)}(\alpha)=d>\pi_{1}^{(n)}(\alpha)$ is symmetric.

LEMMA 5 - Let $C_{0} \geq 1$ a constant and a non trivial non empty subset $\mathcal{A}_{0} \subset \mathcal{A}$, $\mathcal{A}_{0} \neq \mathcal{A}$, such that

$$
\begin{aligned}
& \max _{\alpha \in \mathcal{A}_{0}} Q_{\alpha}(\gamma) \leq C_{0} \min _{\alpha \in \mathcal{A}_{0}} Q_{\alpha}(\gamma), \\
& \max _{\alpha \in \mathcal{A}} Q_{\alpha}(\gamma) \leq \max _{\alpha \in \mathcal{A}_{0}} Q_{\alpha}(\gamma) .
\end{aligned}
$$

There exist a constant $C_{1} \geq 1$, a constant $c_{1} \in(0,1)$, depending only on $C_{0}$ and $d$, and paths $(\gamma(l))_{1 \leq l \leq L}$ extending $\gamma$ such that

(i) the simplices $\Delta(\gamma(l))$ have disjoint interiors and

$$
\operatorname{vol}_{d-1}(\sqcup \Delta(\gamma(l))) \geq c_{1} \operatorname{vol}_{d-1}(\Delta(\gamma)) ;
$$

(ii) for every $l \in[1, L]$, there exists a subset $\mathcal{A}_{l}$ of $\mathcal{A}$ strictly larger than $\mathcal{A}_{0}$ such that

$$
\begin{aligned}
& \max _{\mathcal{A}_{l}} Q_{\alpha}(\gamma(l)) \leq C_{1} \min _{\mathcal{A}_{l}} Q_{\alpha}(\gamma(l)), \\
& \max _{\mathcal{A}} Q_{\alpha}(\gamma(l)) \leq \max _{\mathcal{A}_{l}} Q_{\alpha}(\gamma(l))
\end{aligned}
$$

Proof - We first extend $\gamma$ to a path $\tilde{\gamma}$ such that the name of the last arrow of $\tilde{\gamma}$ does not belong to $\mathcal{A}_{0}$; we can do this having 


$$
\begin{aligned}
& \max _{\mathcal{A}} Q_{\alpha}(\tilde{\gamma}) \leq C_{1}^{\prime} \max _{\mathcal{A}} Q_{\alpha}(\gamma), \\
& \operatorname{vol}_{d-1}(\Delta(\tilde{\gamma})) \geq c_{1}^{\prime} \operatorname{vol}_{d-1}(\Delta(\gamma)),
\end{aligned}
$$

$C_{1}^{\prime}, c_{1}^{\prime}$ depend only on $d$.

We then apply Lemma 4 , for every $\alpha \in \mathcal{A}_{0}$, to $\tilde{\gamma}$ with $C=2 \# \mathcal{A}_{0}$. We obtain that the volume of those $T \in \Delta(\tilde{\gamma})$ for which $Q_{\alpha}^{\prime}(T) \leq 2 \# \mathcal{A}_{0} Q_{\alpha}(\tilde{\gamma})$ for every $\alpha \in \mathcal{A}_{0}$ is at least half the volume of $\Delta(\tilde{\gamma})$. For such a $T$, let $m>\tilde{n}=$ length $(\tilde{\gamma})$ the smallest integer such that the name $\bar{\alpha}$ of $\gamma^{(m)}(T)$ belongs to $\mathcal{A}_{0}$. We define (for those $T$ ) a finite path $\gamma(T)$ as follows :

1. If for some $\tilde{m} \in(\tilde{n}, m)$, some $\alpha \in \mathcal{A}-\mathcal{A}_{0}$, we have

$$
Q_{\alpha}(\tilde{m}, T) \geq \max Q_{\alpha}(\tilde{\gamma})
$$

we let $\gamma(T)=\left(\gamma^{(i)}(T)\right)_{0 \leq i \leq \tilde{m}}$, where $\tilde{m}$ is the smallest such integer.

2. Otherwise, $\gamma(T)=\left(\gamma^{(i)}(T)\right)_{0 \leq i \leq m}$.

We select finitely many such $T_{1}, \ldots, T_{L}$ such that, setting $\gamma(l)=\gamma\left(T_{l}\right)$, we have

$$
\operatorname{vol}(\cup \Delta(\gamma(l))) \geq \frac{1}{4} \operatorname{vol} \Delta(\tilde{\gamma})
$$

and the $\Delta(\gamma(l))$ have disjoint interiors. Let $l \in[1, L]$; if $T_{l}$ is as in case a), we take $\mathcal{A}_{l}$ to be the union of $\mathcal{A}_{0}$ and all $\alpha \in \mathcal{A}-\mathcal{A}_{0}$ satisfying $Q_{\alpha}\left(\tilde{m}, T_{l}\right) \geq \max _{\alpha} Q_{\alpha}(\tilde{\gamma})$. If $T_{l}$ is as in case b), by definition of $m$, the name $\beta$ of $\gamma^{(m-1)}\left(T_{l}\right)$ does not belong to $\mathcal{A}_{0}$ and we have

$$
Q_{\beta}^{(m)}\left(T_{l}\right)=Q_{\alpha}^{(m-1)}\left(T_{l}\right)+Q_{\beta}^{(m-1)}\left(T_{l}\right),
$$

where $\alpha$ is the name of $\gamma^{(m-1)}\left(T_{l}\right)$. It follows that

$$
Q_{\beta}^{(m)}\left(T_{l}\right) \geq C_{0}^{-1} \max _{\alpha \in \mathcal{A}_{0}} Q_{\alpha}(\gamma)
$$

We take $\mathcal{A}_{l}=\mathcal{A}_{0} \cup\{\beta\}$ in this case. We obtain the conclusions of the lemma with $c_{1}=\frac{1}{4} c_{1}^{\prime}$ and $C_{1}=C_{0}\left(1+2\left(\# \mathcal{A}_{0}\right) C_{1}^{\prime}\right)$.

Iterating Lemma 5, we obtain LEMMA 6 - There exists a constant $C$, depending only on $d$, and paths $(\gamma(l))_{1 \leq l \leq L}$ extending $\gamma$ such that

(i) the simplices $\Delta(\gamma(l))$ have disjoint interiors and

(ii) for every $1 \leq l \leq L$, we have

$$
\operatorname{vol}_{d-1}(\sqcup \Delta(\gamma(l))) \geq C^{-1} \operatorname{vol}(\Delta(\gamma)) ;
$$

$$
\left.\max _{\alpha} Q_{\alpha}(\gamma(l))\right) \leq C \min _{\alpha}\left(Q_{\alpha}(\gamma(l))\right)
$$


The proof of the theorem is now clear : for almost every i.e.m $T$, with associated path $\left(\gamma^{(i)}\right)_{i>0}$, it follows from Lemma 6 that there are infinitely many integers $n_{k}$ such that the path $\left(\gamma^{(i)}\right)_{0<i \leq n_{k}}$ satisfy the hypothesis of Lemma 2. It follows then from Lemma 2 that the intersection of the simplices $\Delta\left(\left(\gamma^{(i)}\right)_{0<i \leq n}\right)$ is reduced to a point.

\section{Suspension of i.e.m}

\subsection{Suspension data}

Let $\left(\mathcal{A}, \pi_{0}, \pi_{1}\right)$ be admissible combinatorial data, and let $T$ be an i.e.m of this combinatorial type, determined by length data $\left(\lambda_{\alpha}\right)_{\alpha \in \mathcal{A}}$.

We will construct a Riemann surface with a flow which can be considered as a suspension of $T$. In order to do this, we need data which we call suspension data.

We will identify $\mathbf{R}^{2}$ with $\mathbf{C}$. Consider a family $\tau=\left(\tau_{\alpha}\right)_{\alpha \in \mathcal{A}} \in \mathbf{R}^{\mathcal{A}}$. To this family we associate

$$
\begin{aligned}
& \zeta_{\alpha}=\lambda_{\alpha}+i \tau_{\alpha}, \quad \alpha \in \mathcal{A} \\
& \xi_{\alpha}^{\varepsilon}=\sum_{\pi_{\varepsilon} \beta \leq \pi_{\varepsilon} \alpha} \zeta_{\beta}, \alpha \in \mathcal{A}, \varepsilon \in\{0,1\} .
\end{aligned}
$$

We always have $\xi_{\alpha_{0}}^{0}=\xi_{\alpha_{1}}^{1}$, where as before $\pi_{\varepsilon}\left(\alpha_{\varepsilon}\right)=d$. We say that $\tau$ defines suspension data if the following inequalities hold :

$$
\begin{aligned}
& \operatorname{Im} \xi_{\alpha}^{0}>0 \text { for all } \alpha \in \mathcal{A}, \alpha \neq \alpha_{0}, \\
& \operatorname{Im} \xi_{\alpha}^{1}<0 \text { for all } \alpha \in \mathcal{A}, \alpha \neq \alpha_{1} .
\end{aligned}
$$

We also set

$$
\theta_{\alpha}=\xi_{\alpha}^{1}-\xi_{\alpha}^{0}, \alpha \in \mathcal{A}
$$

We then have

$$
\begin{aligned}
& \theta=\Omega \zeta, \\
& \operatorname{Re} \theta=\delta,
\end{aligned}
$$

and define $h=-\operatorname{Im} \theta=-\Omega \tau$.

One has $h_{\alpha}>0$ for all $\alpha \in \mathcal{A}$, because of the formula

$$
\theta_{\alpha}=\left(\xi_{\alpha}^{1}-\zeta_{\alpha}\right)-\left(\xi_{\alpha}^{0}-\zeta_{\alpha}\right)
$$

One has also 


$$
\operatorname{Im} \xi_{\alpha_{0}}^{0}=\operatorname{Im} \xi_{\alpha_{1}}^{1} \in\left[-h_{\alpha_{1}}, h_{\alpha_{0}}\right]
$$

\subsection{Construction of a Riemann surface}

Let $\left(\mathcal{A}, \pi_{0}, \pi_{1}\right)$ and $\left(\zeta_{\alpha}=\lambda_{\alpha}+i \tau_{\alpha}\right)_{\alpha \in \mathcal{A}}$ as above. For $\alpha \in \mathcal{A}$, consider the rectangles in $\mathbf{C}=\mathbf{R}^{2}$ :

$$
\begin{aligned}
& R_{\alpha}^{0}=\left(R e \xi_{\alpha}^{0}-\lambda_{\alpha}, R e \xi_{\alpha}^{0}\right) \times\left[0, h_{\alpha}\right], \\
& R_{\alpha}^{1}=\left(R e \xi_{\alpha}^{1}-\lambda_{\alpha}, R e \xi_{\alpha}^{1}\right) \times\left[-h_{\alpha}, 0\right],
\end{aligned}
$$

and the segments

$$
\begin{aligned}
& S_{\alpha}^{0}=\left\{\operatorname{Re} \xi_{\alpha}^{0}\right\} \times\left[0, \operatorname{Im} \xi_{\alpha}^{0}\right), \alpha \neq \alpha_{0} \\
& S_{\alpha}^{1}=\left\{\operatorname{Re} \xi_{\alpha}^{1}\right\} \times\left(\operatorname{Im} \xi_{\alpha}^{1}, 0\right], \alpha \neq \alpha_{1} .
\end{aligned}
$$

Let also $S_{\alpha_{0}}^{0}=S_{\alpha_{1}}^{1}$ be the half-open vertical segment $\left[\lambda^{*}, \xi_{\alpha_{0}}^{0}\right)=\left[\lambda^{*}, \xi_{\alpha_{1}}^{1}\right)$. Define then

$$
R_{\zeta}=\bigcup_{\varepsilon} \bigcup_{\alpha} R_{\alpha}^{\varepsilon} \bigcup_{\varepsilon} \bigcup_{\alpha} S_{\alpha}^{\varepsilon} .
$$

The translation by $\theta_{\alpha}$ sends $R_{\alpha}^{0}$ onto $R_{\alpha}^{1}$. If $\xi_{\alpha_{0}}^{0}=\xi_{\alpha_{1}}^{1}=0, S_{\alpha_{0}}^{0}=S_{\alpha_{1}}^{1}$ is empty, $\xi_{\alpha_{1}}^{0}$ is the top right corner of $R_{\alpha_{1}}^{0}$ and $\xi_{\alpha_{0}}^{1}$ is the bottom right corner of $R_{\alpha_{0}}^{1}$. If $\xi_{\alpha_{0}}^{0}=\xi_{\alpha_{1}}^{1}>0$, the translation by $\theta_{\alpha_{1}}$ sends the top part $\tilde{S}_{\alpha_{1}}^{0}=$ $\left\{\operatorname{Re} \xi_{\alpha_{1}}^{0}\right\} \times\left[h_{\alpha_{1}}, \operatorname{Im} \xi_{\alpha_{1}}^{0}\right)$ of $S_{\alpha_{1}}^{0}$ onto $S_{\alpha_{1}}^{1}$. If $\xi_{\alpha_{0}}^{0}=\xi_{\alpha_{1}}^{1}<0$, the translation by $\theta_{\alpha_{0}}$ sends $S_{\alpha_{0}}^{0}$ onto the bottom part $\tilde{S}_{\alpha_{0}}^{1}=\left\{\operatorname{Re} \xi_{\alpha_{0}}^{1}\right\} \times\left(\operatorname{Im} \xi_{\alpha_{0}}^{1},-h_{\alpha_{0}}\right]$ of $S_{\alpha_{0}}^{1}$.

We use these translations to identify in $R_{\zeta}$ each $R_{\alpha}^{0}$ to each $R_{\alpha}^{1}$, and $S_{\alpha_{0}}^{0}=S_{\alpha_{1}}^{1}$ (if non empty) to either $\tilde{S}_{\alpha_{1}}^{0}$ or $\tilde{S}_{\alpha_{0}}^{1}$. Denote by $M_{\zeta}^{*}$ the topological space obtained from $R_{\zeta}$ by these identifications.

Observe that $M_{\zeta}^{*}$ inherits from $\mathbf{C}$ the structure of a Riemann surface, and also a nowhere vanishing holomorphic 1 -form $\omega$ (given by $d z$ ) and a vertical vector field (given by $\frac{\partial}{\partial y}$ ).

\subsection{Compactification of $M_{\zeta}^{*}$}

Let $\overline{\mathcal{A}}$ be the set with $2 d-2$ elements of pairs $(\alpha, L)$ and $(\alpha, R)$, except that we identify $\left(\alpha_{0}, R\right)=\left(\alpha_{1}, R\right)$ and $\left(\alpha_{0}^{\prime}, L\right)=\left(\alpha_{1}^{\prime}, L\right)$, where $\pi_{\varepsilon}\left(\alpha_{\varepsilon}\right)=d, \pi_{\varepsilon}\left(\alpha_{\varepsilon}^{\prime}\right)=$ 1.

Let $\sigma$ be the permutation of $\overline{\mathcal{A}}$ defined by

$$
\begin{aligned}
& \sigma(\alpha, R)=\left(\beta_{0}, L\right), \\
& \sigma(\alpha, L)=\left(\beta_{1}, R\right),
\end{aligned}
$$

with $\pi_{0}\left(\beta_{0}\right)=\pi_{0}(\alpha)+1, \pi_{1}\left(\beta_{1}\right)=\pi_{1}(\alpha)-1$; in particular, we have 


$$
\begin{aligned}
& \sigma\left(\alpha_{0}, R\right)=\left(\pi_{0}^{-1}\left(\pi_{0}\left(\alpha_{1}\right)+1\right), L\right), \\
& \sigma\left(\alpha_{1}^{\prime}, L\right)=\left(\pi_{1}^{-1}\left(\pi_{1}\left(\alpha_{0}^{\prime}\right)-1\right), R\right) .
\end{aligned}
$$

The permutation describes which half planes are met when one winds around an end of $M_{\zeta}^{*}$. Denote by $\sum$ the set of cycles of $\sigma$. To each $c \in \Sigma$ is associated in a one-to-one correspondance an end $q_{c}$ of $M_{\zeta}^{*}$. From the local structure around $q_{c}$, it is clear that the compactification $M_{\zeta}=M_{\zeta}^{*} \bigcup_{\Sigma}\left\{q_{c}\right\}$ will be a compact Riemann surface, with the set of marked points $\left\{q_{c}\right\}=M_{\zeta}-M_{\zeta}^{*}$ in canonical correspondence with $\Sigma$. Moreover, the 1 -form $\omega$ extends to a holomorphic 1-form on $M_{\zeta}$; the length of a cycle $c$ is an even number $2 n_{c}$; the corresponding marked point $q_{c}$ is a zero of $\omega$ of order $n_{c}-1$.

Let $\nu=\# \Sigma$, and let $g$ be the genus of $M_{\zeta}$. We have

$$
\begin{aligned}
& d-1=\Sigma n_{c} \\
& 2 g-2=\Sigma\left(n_{c}-1\right)
\end{aligned}
$$

hence

$$
d=2 g+\nu-1
$$

Example : Suppose that $\pi_{0}, \pi_{1}$ satisfy

$$
\pi_{0}(\alpha)+\pi_{1}(\alpha)=d+1, \text { for all } \alpha \in \mathcal{A}
$$

If $d$ is even, there is only 1 cycle ; we have $d=2 g$ and the only zero of $\omega$ has order $2 g-2$. If $d$ is odd, there are two cycles of equal length $d-1$; we have $d=2 g+1$, and each of the two zeros of $\omega$ has order $g-1$.

The vertical vector field on $M_{\zeta}^{*}$ does not extend (continuously) to $M_{\zeta}$ when $g>1$, unless one slows it near the marked points (which we will not do here). Nevertheless, it can be considered as a suspension of $T:$ starting from a point $(x, 0)$ on the bottom side of $R_{\alpha}^{0}$, one flows up till reaching the top side where the point $\left(x, h_{\alpha}\right)$ is identified with the point $\left(x+\delta_{\alpha}, 0\right)=(T(x), 0)$ in the top side of $R_{\alpha}^{1}$. The return time is $h_{\alpha}$. The vector field is not complete, as some orbits reach marked points in finite time.

\subsection{The basic operation of the algorithm for suspensions}

Let $\left(\mathcal{A}, \pi_{0}, \pi_{1}\right)$ and $\left(\zeta_{\alpha}=\lambda_{\alpha}+i \tau_{\alpha}\right)_{\alpha \in \mathcal{A}}$ as above. Construct $R_{\zeta}, M_{\zeta}$ as in 5.2 , 5.3. With $\pi_{\varepsilon}\left(\alpha_{\varepsilon}\right)=d$ as above, assume that

$$
\lambda_{\alpha_{0}} \neq \lambda_{\alpha_{1}}
$$

Then the formula $\lambda_{\alpha_{\varepsilon}}=\max \left(\lambda_{\alpha_{0}}, \lambda_{\alpha_{1}}\right)$ defines uniquely $\varepsilon \in\{0,1\}$ and determines uniquely the basic step of the continuous fraction algorithm; this step produces new combinatorial data $\left(\mathcal{A}, \hat{\pi}_{0}, \hat{\pi}_{1}\right)$ and length data $\left(\hat{\lambda}_{\alpha}\right)_{\alpha \in \mathcal{A}}$ given by 


$$
\left\{\begin{array}{l}
\hat{\lambda}_{\alpha}=\lambda_{\alpha}, \quad \alpha \neq \alpha_{\varepsilon} \\
\hat{\lambda}_{\alpha_{\varepsilon}}=\lambda_{\alpha_{\varepsilon}}-\lambda_{\alpha_{1-\varepsilon}}
\end{array}\right.
$$

For suspension data, we just define in the same way

$$
\left\{\begin{array}{l}
\hat{\zeta}_{\alpha}=\zeta_{\alpha}, \quad \alpha \neq \alpha_{\varepsilon} \\
\hat{\zeta}_{\alpha_{\varepsilon}}=\zeta_{\alpha_{\varepsilon}}-\zeta_{\alpha_{1-\varepsilon}}
\end{array}\right.
$$

This has a nice representation in terms of the corresponding regions $R_{\zeta}, R_{\hat{\zeta}}$. One cuts from $R_{\zeta}$ the part where $x>\hat{\lambda}^{*}=\lambda^{*}-\lambda_{\alpha_{\varepsilon}}$; it is made of $R_{\alpha_{1-\varepsilon}}^{1-\varepsilon}$ and a right part of $R_{\alpha_{\varepsilon}}^{\varepsilon}$. We glue back $R_{\alpha_{1-\varepsilon}}^{1-\varepsilon}$ to the free horizontal side of $R_{\alpha_{\varepsilon}}^{1-\varepsilon}$, and the right part of $R_{\alpha_{\varepsilon}}^{\varepsilon}$ to $R_{\alpha_{1-\varepsilon}}^{\varepsilon}$ : see figure 5 .

It is easy to check that the new suspension data satisfy the inequalities required in 5.1 ; if for instance $\varepsilon=0$, one has

$$
\hat{\xi}_{\alpha}^{0}=\xi_{\alpha}^{0}, \alpha \neq \alpha_{0}
$$

with $\hat{\pi}_{0}=\pi_{0}$ on one hand and

$$
\begin{aligned}
& \hat{\xi}_{\alpha}^{1}=\xi_{\alpha}^{1}, \quad \alpha \neq \alpha_{0}, \alpha_{1} \\
& \hat{\xi}_{\alpha_{1}}^{1}=\xi_{\alpha_{0}}^{1}, \\
& \hat{\xi}_{\alpha_{0}}^{1}=\xi_{\alpha_{0}}^{1}-\zeta_{\alpha_{1}} .
\end{aligned}
$$

The last formula gives

$$
\begin{aligned}
-\hat{\xi}_{\alpha_{0}}^{1} & =\zeta_{\alpha_{1}}-\xi_{\alpha_{0}}^{1} \\
& =\zeta_{\alpha_{1}}-\xi_{\alpha_{0}}^{0}-\theta_{\alpha_{0}} \\
& =\zeta_{\alpha_{1}}-\xi_{\alpha_{1}}^{1}-\theta_{\alpha_{0}} \\
& =-\xi_{\tilde{\alpha}_{1}}^{1}-\theta_{\alpha_{0}},
\end{aligned}
$$

with $\pi_{1}\left(\tilde{\alpha}_{1}\right)=d-1$. We therefore have

$$
-\operatorname{Im} \hat{\xi}_{\alpha_{0}}^{1}=-\operatorname{Im} \xi_{\tilde{\alpha}_{1}}^{1}+h_{\alpha}>0 . .
$$

We also see that (still with $\varepsilon=0$ ), if $\hat{\alpha}_{1} \in \mathcal{A}$ is such that $\hat{\pi}_{1}\left(\hat{\alpha}_{1}\right)=d$ (we have $\hat{\alpha}_{1}=\tilde{\alpha}_{1}$ if $\tilde{\alpha}_{1} \neq \alpha_{0}, \hat{\alpha}_{1}=\alpha_{1}$ if $\tilde{\alpha}_{1}=\alpha_{0}$ ), one has

$$
\operatorname{Im} \hat{\xi}_{\hat{\alpha}_{1}}^{1}=\operatorname{Im} \xi_{\tilde{\alpha}_{1}}^{1}<0
$$

Conversely, given $\left(\mathcal{A}, \pi_{0}, \pi_{1}\right)$ and $\left(\zeta_{\alpha}=\lambda_{\alpha}+i \tau_{\alpha}\right)_{\alpha \in \mathcal{A}}$ as above, assume that

$$
\operatorname{Im} \xi_{\alpha_{0}}^{0}=\operatorname{Im} \xi_{\alpha_{1}}^{1} \neq 0
$$

and define $\varepsilon$ as 0 if $\operatorname{Im} \xi_{\alpha_{1}}^{1}<0,1$ if $\operatorname{Im} \xi_{\alpha_{0}}^{0}>0$. Set 

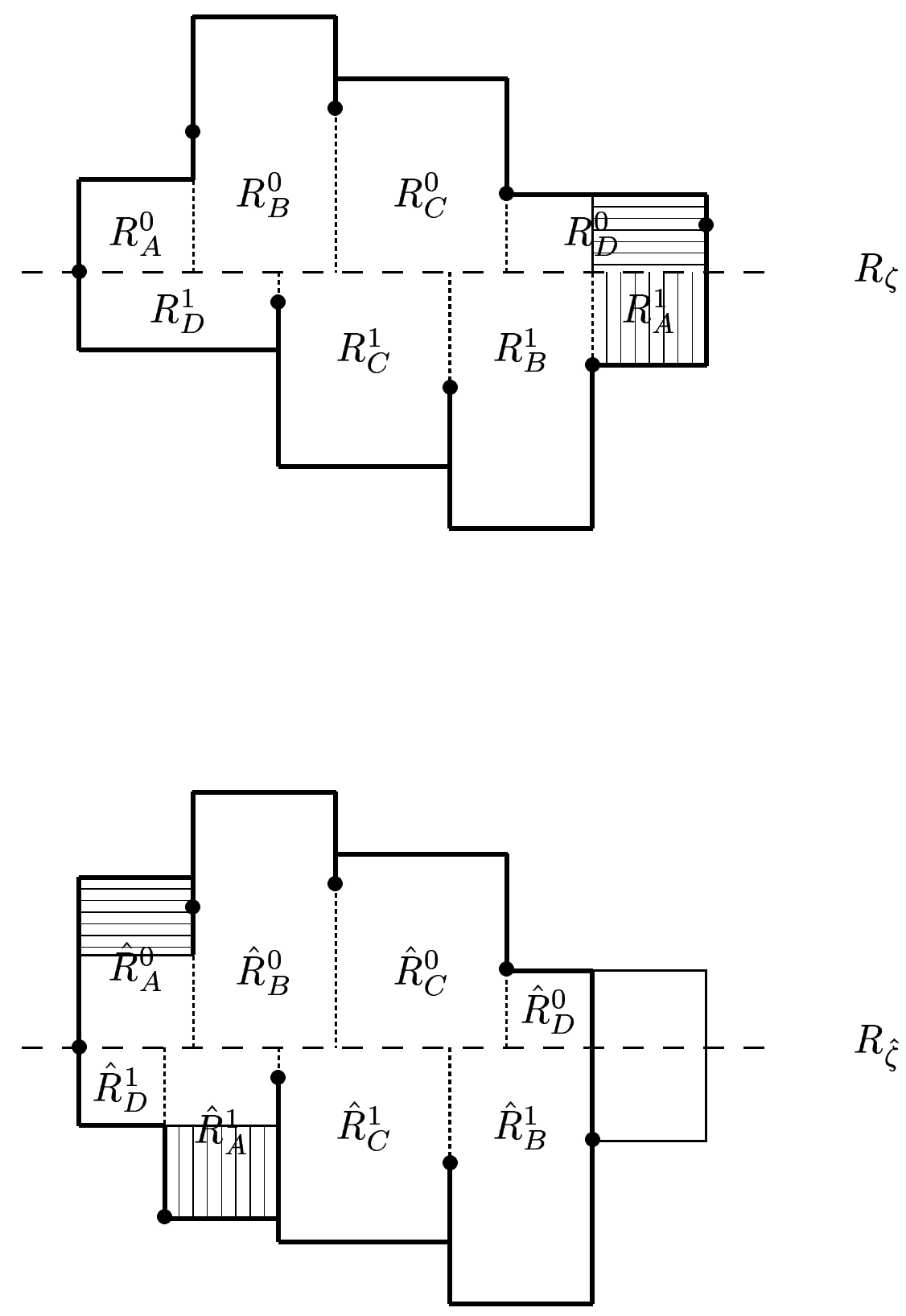

Fig. 5. The Rauzy-Veech operation for suspensions 


$$
\left\{\begin{array}{l}
\hat{\zeta}_{\alpha}=\zeta_{\alpha} \text { for } \alpha \neq \alpha_{\varepsilon}, \\
\hat{\zeta}_{\alpha_{\varepsilon}}=\zeta_{\alpha_{\varepsilon}}+\zeta_{\alpha_{1-\varepsilon}}
\end{array}\right.
$$

and define appropriately new combinatorial data ; this operation is the inverse of the one above. Thus the dynamics of the continuous fraction algorithm at the level of suspensions is invertible (on a full measure set) and can be viewed as the natural extension of the dynamics at the level of i.e.m.

It is clear that the Riemann surfaces $M_{\zeta}, M_{\hat{\zeta}}$ are canonically isomorphic, and the isomorphism respects the holomorphic 1 -form and the vertical vector field.

\subsection{Cohomological interpretation of $\Omega$}

Consider the following homology classes :

- $c_{\alpha} \in H_{1}\left(M_{\zeta}, \Sigma, \mathbf{Z}\right)$ is defined by a path in $R_{\zeta}$ joining $\xi_{\alpha}^{0}-\zeta_{\alpha}$ to $\xi_{\alpha}^{0}$ (or by a path joining $\xi_{\alpha}^{1}-\zeta_{\alpha}$ to $\left.\xi_{\alpha}^{1}\right)$;

- $c_{\alpha}^{*} \in H_{1}\left(M_{\zeta}-\Sigma, \mathbf{Z}\right)$ is defined by a path in $R_{\zeta}$ joining the center of $R_{\alpha}^{0}$ to the center of $R_{\alpha}^{1}$.

Then $\left(c_{\alpha}\right)_{\alpha \in \mathcal{A}}$ is a basis of $H_{1}\left(M_{\zeta}, \Sigma, \mathbf{Z}\right)$, and $\left(c_{\alpha}^{*}\right)_{\alpha \in \mathcal{A}}$ is a basis of $H_{1}\left(M_{\zeta}-\Sigma, \mathbf{Z}\right)$.

For the intersection pairing on $H_{1}\left(M_{\zeta}-\Sigma, \mathbf{Z}\right) \times H_{1}\left(M_{\zeta}, \Sigma, \mathbf{Z}\right),\left(c_{\alpha}^{*}\right)$ and $\left(c_{\alpha}\right)$ are dual bases.

We have canonical maps

$$
H_{1}\left(M_{\zeta}-\Sigma, \mathbf{Z}\right) \rightarrow H_{1}\left(M_{\zeta}, \mathbf{Z}\right) \rightarrow H_{1}\left(M_{\zeta}, \Sigma, \mathbf{Z}\right)
$$

where the first map is surjective and the second injective ; the image of $c_{\alpha}^{*}$ in $H_{1}\left(M_{\zeta}, \Sigma, \mathbf{Z}\right)$ is equal to $\sum_{\beta} \Omega_{\alpha \beta} c_{\beta}$.

The 1-form $\omega$ determines a cohomology class $[\omega]$ in $H^{1}\left(M_{\zeta}, \Sigma, \mathbf{C}\right)$ : we have

$$
\int_{c_{\alpha}} \omega=\zeta_{\alpha}
$$

We have the dual sequence

$$
H^{1}\left(M_{\zeta}, \Sigma, \mathbf{C}\right) \rightarrow H^{1}\left(M_{\zeta}, \mathbf{C}\right) \rightarrow H^{1}\left(M_{\zeta}-\Sigma, \mathbf{C}\right)
$$

where the first map is surjective and the second injective. The image of $[\omega]$ in $H^{1}\left(M_{\zeta}-\Sigma, \mathbf{C}\right)$ satisfies 


$$
\int_{c_{\alpha}^{*}} \omega=\theta_{\alpha}=(\Omega \zeta)_{\alpha}
$$

Thus $\Omega$ is the matrix of the composition

$$
H^{1}\left(M_{\zeta}, \Sigma, \mathbf{C}\right) \rightarrow H^{1}\left(M_{\zeta}-\Sigma, \mathbf{C}\right)
$$

The image of $\Omega$ is equal to the image of $H^{1}\left(M_{\zeta}, \mathbf{C}\right)$ into $H^{1}\left(M_{\zeta}-\Sigma, \mathbf{C}\right)$.

When one performs the basic operation of the continuous fraction algorithm and one identifies $M_{\zeta}$ with $M_{\hat{\zeta}}$, the relation between the old and new bases is given by

$$
\begin{gathered}
\left\{\begin{array}{l}
\hat{c}_{\alpha}=c_{\alpha} \text { if } \alpha \neq \alpha_{\varepsilon}, \\
\hat{c}_{\alpha_{\varepsilon}}=c_{\alpha_{\varepsilon}}-c_{\alpha_{1-\varepsilon}}
\end{array}\right. \\
\left\{\begin{array}{l}
\hat{c}_{\alpha}^{*}=c_{\alpha}^{*} \text { if } \alpha \neq \alpha_{1-\varepsilon} \\
\hat{c}_{\alpha_{1-\varepsilon}}^{*}=c_{\alpha_{1-\varepsilon}}^{*}+c_{\alpha_{\varepsilon}}^{*}
\end{array}\right.
\end{gathered}
$$

At the cohomological level, we have an isomorphism of $H^{1}\left(M_{\zeta}, \mathbf{C}\right)$ given by

$$
\left\{\begin{array}{l}
\hat{\theta}_{\alpha}=\theta_{\alpha} \text { if } \alpha \neq \alpha_{1-\varepsilon} \\
\hat{\theta}_{\alpha_{1-\varepsilon}}=\theta_{\alpha_{1-\varepsilon}}+\theta_{\alpha_{\varepsilon}}
\end{array}\right.
$$

(these formulas determine an isomorphism from $\operatorname{Im} \Omega$ onto $\operatorname{Im} \hat{\Omega}$ ). This is the discrete version of the so-called Kontsevich-Zorich cocycle.

\subsection{The Teichmüller flow}

Fix combinatorial data $\left(\mathcal{A}, \pi_{0}, \pi_{1}\right)$. Given length data $\left(\lambda_{\alpha}\right)$ and suspension data $\left(\tau_{\alpha}\right)$, one defines for $t \in \mathbf{R}$

$$
U^{t}(\lambda, \tau)=\left(e^{t / 2} \lambda, e^{-t / 2} \tau\right)
$$

This flow is called the Teichmüller flow. Observe that the conditions on the length data $\left(\lambda_{\alpha}>0\right)$ and on the suspension data (cf. 4.1) are preserved under the flow.

It is also obvious that the flow commutes with the basic operation of the continuous fraction algorithm. In particular, the inequality $\lambda_{\alpha_{\varepsilon}}>\lambda_{\alpha_{1-\varepsilon}}$ is preserved.

The surface $M_{\zeta}$ is canonically equipped with an area form (coming from C) for which its area is

$$
A:=\operatorname{area}\left(M_{\zeta}\right)=\sum_{\alpha \in \mathcal{A}} \lambda_{\alpha} h_{\alpha} .
$$


The area is preserved by the Teichmüller flow, and also by the basic operation of the continuous fraction algorithm.

The Lebesgue measure $d \lambda d \tau$ on the domain of $\mathbf{R}^{\mathcal{A}} \times \mathbf{R}^{\mathcal{A}}$ defined by the restrictions on length and suspension data is preserved by the Teichmüller flow, and by the basic operation of the continuous fraction algorithm.

\section{Invariant measures}

\subsection{The case $d=2$}

We have seen in 2.6 that i.e.m in this case are just rotations on the circle.

Let $\left(\lambda_{A}, \lambda_{B}\right)$ be the length data. The basic step of the continuous fraction algorithm sends these data on $\left(\lambda_{A}-\lambda_{B}, \lambda_{B}\right)$ (resp. $\left.\left(\lambda_{A}, \lambda_{B}-\lambda_{A}\right)\right)$ if $\lambda_{A}>\lambda_{B}$ (resp. $\left.\lambda_{A}<\lambda_{B}\right)$. Set $x=\lambda_{B} / \lambda_{A}$ if $\lambda_{B}<\lambda_{A}, x=\lambda_{A} / \lambda_{B}$ if $\lambda_{A}<\lambda_{B}$. We obtain the well-known map

$$
g(x)=\left\{\begin{array}{l}
\frac{x}{1-x} \text { for } 0<x<1 / 2 \\
g(1-x)=\frac{1-x}{x} \text { for } 1 / 2<x<1,
\end{array}\right.
$$

with a parabolic fixed point at 0 . This map has $\frac{d x}{x}$ as a unique (up to a multiplicative constant) invariant measure absolutely continuous w.r.t Lebesgue measure, but this measure is infinite!

Instead, the Gauss map

$$
G(x)=\left\{x^{-1}\right\}
$$

has $\frac{d x}{1+x}$ as a unique (up to a multiplicative constant) invariant measure absolutely continuous w.r.t Lebesgue measure, but the density is now analytic on $[0,1]$.

The map $G$ is related to $g$ as follows : we have $G(x)=g^{n}(x)$, where $n$ is the smallest integer $>0$ such that $g^{n-1}(x) \in[1 / 2,1)$.

For a general Rauzy diagram (with admissible combinatorial data), Veech has shown ([V2]) that there exists a unique (up to a multiplicative constant) measure absolutely continuous w.r.t Lebesgue measure which is invariant under the normalized continuous fraction algorithm. But again, this measure is infinite.

Following Zorich, it is however possible to accelerate the Rauzy-Veech algorithm, concatenating several successive steps in a single one (as the Gauss map does). For the new algorithm, there will exist an invariant absolutely continuous probability measure, which is very useful for ergodic - theoretic considerations.

\subsection{The accelerated algorithm $([\mathrm{Z} 1])$}


Let $\left(\mathcal{A}, \pi_{0}, \pi_{1}\right)$ be admissible combinatorial data and $\left(\lambda_{\alpha}\right)_{\alpha \in \mathcal{A}}$ be length data. Assume for simplicity that the i.e.m $T$ defined by these data satisfies the Keane property.

The continuous fraction algorithm applied to $T$ gives an infinite path in the Rauzy diagram of $\left(\mathcal{A}, \pi_{0}, \pi_{1}\right)$, starting at the vertex $\left(\pi_{0}, \pi_{1}\right)$, that we denote by $\left(\gamma_{n}(T)\right)_{n>0}$. To each arrow $\gamma_{n}$ is associated a type (0 or 1$)$ and a name (a letter in $\mathcal{A})$; it is obvious from the definitions of type and name that $\gamma_{n}, \gamma_{n+1}$ have the same type iff they have the same name. We also know that each name is taken infinitely many times (proposition in 4.3); the same assertion for types is actually obvious.

In the accelerated algorithm, one performs in a single step the consecutive steps of the (slow) algorithm for which the associated arrows have the same type (or name).

Assume for instance that $\lambda_{\alpha_{0}}>\lambda_{\alpha_{1}}$. Write $\pi_{1}\left(\alpha_{0}\right)=d-\bar{d}<d$ and $\pi_{1}\left(\alpha_{1}^{(i)}\right)=d-i$ for $0 \leq i<\bar{d}$. The accelerated algorithm makes the following "euclidean division" : one substracts from $\lambda_{\alpha_{0}}$ in turn $\lambda_{\alpha_{1}^{(0)}}, \lambda_{\alpha_{1}^{(1)}}, \ldots \lambda_{\alpha_{1}^{(\bar{d}-1)}}$, $\lambda_{\alpha_{1}^{(0)}}, \lambda_{\alpha_{1}^{(1)}} \ldots$ stopping just before the result becomes negative. This is a single step for the accelerated algorithm. When $\bar{d}=1$, for instance when $d=2$, it just amounts to ordinary euclidean division with remainder.

We can extend the definition of the accelerated algorithm at the level of suspension data. Recall that at this level, the dynamics of the slow algorithm are essentially invertible (i.e modulo a set of codimension one). The dynamics of the accelerated algorithm is a first return map of the dynamics of the slow one. Indeed, for fixed combinatorial data $\left(\mathcal{A}, \pi_{0}, \pi_{1}\right)$, the simplicial cone of length data is divided into the two simplicial subcones $\left\{\lambda_{\alpha_{0}}>\lambda_{\alpha_{1}}\right\}$ and $\left\{\lambda_{\alpha_{1}}>\lambda_{\alpha_{0}}\right\}$ according to the type 0 or 1 of the basic step. On the other hand, we have seen in 5.4 that the polyhedral cone of suspension data is divided into $\left\{\operatorname{Im} \xi_{\alpha_{1}}^{1}<0\right\}$ and $\left\{\operatorname{Im} \xi_{\alpha_{0}}^{0}>0\right\}$ according to the type 0 or 1 of the prior basic step.

Therefore, we set

$$
\begin{aligned}
& \mathcal{Z}_{0}=\left\{\lambda_{\alpha_{0}}>\lambda_{\alpha_{1}}, \operatorname{Im} \xi_{\alpha_{0}}^{0}>0\right\}, \\
& \mathcal{Z}_{1}=\left\{\lambda_{\alpha_{1}}>\lambda_{\alpha_{0}}, \operatorname{Im} \xi_{\alpha_{1}}^{1}<0\right\}, \\
& \mathcal{Z}=\mathcal{Z}_{0} \sqcup \mathcal{Z}_{1} . .
\end{aligned}
$$

The accelerated algorithm is the first return map to $\mathcal{Z}$ of the slow algorithm.

Till now, we have considered $\lambda^{*}:=\Sigma \lambda_{\alpha}=1$ as the natural normalization for the length data. Actually, in the sequel, a different normalization seems preferable. As in 4.1, for $\lambda_{\alpha_{\varepsilon}}>\lambda_{\alpha_{1-\varepsilon}}$, set

$$
\left\{\begin{array}{l}
\hat{\lambda}_{\alpha}=\lambda_{\alpha} \text { if } \alpha \neq \alpha_{\varepsilon} \\
\hat{\lambda}_{\alpha_{\varepsilon}}=\lambda_{\alpha_{\varepsilon}}-\lambda_{\alpha_{1-\varepsilon}}
\end{array}\right.
$$


Define then $\hat{\lambda}^{*}:=\sum_{\alpha} \hat{\lambda}_{\alpha}=\lambda^{*}-\lambda_{\alpha_{1-\varepsilon}} ;$ we will normalize by $\left\{\hat{\lambda}^{*}=1\right\}$.

\subsection{The absolutely continuous invariant measure}

Consider the accelerated algorithm acting on the region $\mathcal{Z}$ of the $(\lambda, \tau)$ space. It is invertible (up to a codimension one subset) and acts by unimodular matrices. Therefore the restriction $m_{0}$ of Lebesgue measure to $\mathcal{Z}$ is invariant. The area function $A=\sum_{\alpha} \lambda_{\alpha} h_{\alpha}$ is also invariant, where $h=-\Omega \tau$.

We now use the Teichmüller flow $U^{t}$ to have the horizontal length $\hat{\lambda}^{*}$ also invariant. More precisely, let $\left(\pi_{0}, \pi_{1}, \lambda, \tau\right) \in \mathcal{Z}$, with image $\left(\bar{\pi}_{0}, \bar{\pi}_{1}, \bar{\lambda}, \bar{\tau}\right)$ under the accelerated algorithm. Set

$$
\begin{aligned}
& t(\lambda)=2\left(\log \hat{\lambda}^{*}-\log \hat{\bar{\lambda}}^{*}\right), \\
& \bar{G}\left(\pi_{0}, \pi_{1}, \lambda, \tau\right)=\left(\bar{\pi}_{0}, \bar{\pi}_{1}, U^{t(\lambda)}(\bar{\lambda}, \bar{\tau})\right),
\end{aligned}
$$

and call $\bar{G}$ the normalized basic step for (the natural extension of) the accelerated algorithm. The measure $m_{0}$ is still invariant under $\bar{G}$ because $m_{0}$ is invariant under the Teichmüller flow and $t$ is constant along the orbits of the flow. The area function $A$ is still invariant. The length function $\hat{\lambda}^{*}$ is now also invariant by construction. Define

$$
\mathcal{Z}^{(1)}=\mathcal{Z} \cap\{A \leq 1\},
$$

and denote by $m_{1}$ the restriction of $m_{0}$ to $\mathcal{Z}^{(1)}$. We now project to $\mathcal{C}(\mathcal{D})$ (cf. 4.3) : we obtain a map

$$
G\left(\pi_{0}, \pi_{1}, \lambda\right)=\left(\bar{\pi}_{0}, \bar{\pi}_{1}, e^{\frac{1}{2} t(\lambda)} \bar{\lambda}\right)
$$

and a measure $m_{2}$, image of $m_{1}$ by the projection, which is invariant under $G$. As $\hat{\lambda}^{*}$ is still invariant under $G$, we can restrict, by homogeneity, the measure $m_{2}$ to $\left\{\hat{\lambda}^{*}=1\right\}$ and get the measure $m$ invariant under $G$, that we are looking for. We will now check its properties.

\subsection{Computation of a volume}

The density of the measure $m_{2}$ (w.r.t Lebesgue measure in $\lambda$ space) is given by the volume of the fiber of the projection sending $m_{1}$ onto $m_{2}$. Therefore, we have to compute the volumes of

$$
\Gamma_{\varepsilon} \cap\{A \leq 1\}
$$

where

$$
\begin{aligned}
& \Gamma_{0}=\left\{\operatorname{Im} \xi_{\alpha}^{0}>0, \forall \alpha \in \mathcal{A}, \operatorname{Im} \xi_{\alpha}^{1}<0, \forall \alpha \neq \alpha_{1}\right\}, \\
& \Gamma_{1}=\left\{\operatorname{Im} \xi_{\alpha}^{0}>0, \forall \alpha \neq \alpha_{0}, \operatorname{Im} \xi_{\alpha}^{1}<0, \forall \alpha \in \mathcal{A}\right\},
\end{aligned}
$$


and $\lambda_{\alpha_{\varepsilon}}>\lambda_{\alpha_{1-\varepsilon}}$.

The computation is symmetric and we only consider the case $\varepsilon=0$. We write the polyhedral cone $\Gamma_{0}$ in $\tau$-space as a union of finitely many disjoint simplicial cones $\Gamma$ up to a codimension 1 subset ; for each $\Gamma$, we choose a basis $\tau^{(1)}, \ldots, \tau^{(d)}$ of $\mathbf{R}^{\mathcal{A}}$ with volume 1 which generates $\Gamma$ :

$$
\Gamma=\left\{\sum_{j=1}^{d} t_{j} \tau^{(j)}, t_{j} \geq 0\right\} .
$$

We have

$$
\operatorname{vol}_{d}\left(\Gamma \cap\left\{\Sigma \lambda_{\alpha} h_{\alpha} \leq 1\right\}\right)=(d !)^{-1} \prod_{1}^{d}\left(\Sigma \lambda_{\alpha} h_{\alpha}^{(j)}\right)^{-1},
$$

where $h^{(j)}=-\Omega \tau^{(j)}$. This gives for the density $\mathcal{X}$ of $m_{2}$ the formula

$$
(*) \quad \mathcal{X}_{\pi_{0}, \pi_{1}}(\lambda)=(d !)^{-1} \sum_{\varepsilon} \sum_{\Gamma} \prod_{1}^{d}\left(\Sigma \lambda_{\alpha} h_{\alpha}^{(j)}\right)^{-1} .
$$

To estimate further the density, we write, when $\varepsilon=0$ :

$$
\begin{aligned}
& \hat{\lambda}_{\alpha_{0}}=\lambda_{\alpha_{0}}-\lambda_{\alpha_{1}}, \\
& \hat{h}_{\alpha_{1}}=h_{\alpha_{0}}+h_{\alpha_{1}},
\end{aligned}
$$

and $\hat{\lambda}_{\alpha}=\lambda_{\alpha}, \hat{h}_{\alpha}=h_{\alpha}$ otherwise. We have

$$
\sum_{\alpha} \lambda_{\alpha} h_{\alpha}^{(j)}=\sum_{\alpha} \hat{\lambda}_{\alpha} \hat{h}_{\alpha}^{(j)}
$$

and define

$$
W_{j}=\left\{\alpha \in \mathcal{A}, \hat{h}_{\alpha}^{(j)} \neq 0\right\} .
$$

\subsection{The key combinatorial lemma ([V2], [Z1])}

PROPOSITION - Let $X$ be a subset of $\mathcal{A}$, non empty and distinct from $\mathcal{A}$. Let $E_{X}$ be the subspace of $\mathbf{R}^{\mathcal{A}}$ generated by the $\tau \in \Gamma_{0}$ such that $h=-\Omega \tau$ satisfies $\hat{h}_{\alpha}=0$ for all $\alpha \in X$. Then the codimension of $E_{X}$ is $>\# X$.

COROLlary - \#\{j, $\left.W_{j} \cap X=\emptyset\right\}+\# X<d$.

Proof of corollary- One has $W_{j} \cap X=\emptyset$ iff $\tau^{(j)} \in E_{X}$, and the $\tau^{(j)}$ are linearly independent.

Proof of proposition - As usual, we denote by $\alpha_{0}, \alpha_{1}, \alpha_{0}^{\prime}, \alpha_{1}^{\prime}$ the elements such that $\pi_{\varepsilon}\left(\alpha_{\varepsilon}\right)=d, \pi_{\varepsilon}\left(\alpha_{\varepsilon}^{\prime}\right)=1$. We write the $\hat{h}_{\alpha}$ in terms of those $(-1)^{\varepsilon} \operatorname{Im} \xi_{\alpha}^{\varepsilon},(\varepsilon, \alpha)$ which are nonnegative, i.e. with $(\varepsilon, \alpha) \neq\left(1, \alpha_{1}\right)$.

We have 


$$
\hat{h}_{\alpha}=\operatorname{Im} \xi_{\alpha}^{0}-\operatorname{Im} \xi_{\alpha}^{1}=\operatorname{Im} \xi_{\beta_{0}}^{0}-\operatorname{Im} \xi_{\beta_{1}}^{1}
$$

for $\alpha \neq \bar{\alpha}_{0}, \bar{\alpha}_{1}, \alpha_{1}$; we have denoted by $\beta_{0}, \beta_{1}$ the elements such that $\pi_{\varepsilon}\left(\beta_{\varepsilon}\right)=$ $\pi_{\varepsilon}(\alpha)-1$. The same formula still holds for $\alpha=\bar{\alpha}_{1}$ and $\alpha=\bar{\alpha}_{0} \neq \alpha_{1}$, with the convention that $\operatorname{Im} \xi_{\beta_{0}}^{0}=0$ if $\alpha=\bar{\alpha}_{0}$ and $\operatorname{Im} \xi_{\beta_{1}}^{1}=0$ if $\alpha=\bar{\alpha}_{1}$. For $\alpha=\alpha_{1}$, we have

$$
\begin{aligned}
\hat{h}_{\alpha_{1}} & =\operatorname{Im} \xi_{\alpha_{1}}^{0}+\operatorname{Im} \xi_{\alpha_{0}}^{1} \\
& =\operatorname{Im} \xi_{\beta_{0}}^{0}=\operatorname{Im} \xi_{\beta_{1}}^{1}+\operatorname{Im} \xi_{\gamma_{0}}^{0}+\operatorname{Im} \xi_{\gamma_{1}}^{1}
\end{aligned}
$$

with $\pi_{\varepsilon}\left(\beta_{\varepsilon}\right)=\pi_{\varepsilon}\left(\alpha_{1}\right)-1, \pi_{\varepsilon}\left(\gamma_{\varepsilon}\right)=\pi_{\varepsilon}\left(\alpha_{0}\right)-1$.

From these formulas, we define subsets $\mathcal{A}(\varepsilon, \alpha) \subset \mathcal{A}$, (with $\mathcal{A}(1, \alpha) \subset$ $\left.\mathcal{A}-\left\{\alpha_{1}\right\}\right)$ such that $\hat{h}_{\alpha}=0$ implies $\operatorname{Im} \xi_{\beta}^{\varepsilon}=0$ for $\beta \in \mathcal{A}(\varepsilon, \alpha)$ : we have

$$
\begin{array}{ll}
\mathcal{A}(0, \alpha)=\left\{\beta_{0}, \alpha\right\} & \text { if } \alpha \neq \bar{\alpha}_{0}, \alpha_{1}, \\
\mathcal{A}\left(0, \bar{\alpha}_{0}\right)=\left\{\bar{\alpha}_{0}\right\} & \text { if } \bar{\alpha}_{0} \neq \alpha_{1}, \\
\mathcal{A}\left(0, \alpha_{1}\right)=\left\{\gamma_{0}, \beta_{0}, \alpha_{1}\right\}, & \text { if } \alpha \neq \bar{\alpha}_{1}, \alpha_{1}, \\
\mathcal{A}\left(0, \bar{\alpha}_{0}\right)=\mathcal{A}\left(1, \alpha_{1}\right)=\left\{\gamma_{0}, \bar{\alpha}_{0}=\alpha_{1}\right\} & \text { if } \bar{\alpha}_{0}=\alpha_{1} ; \\
\mathcal{A}(1, \alpha)=\left\{\beta_{1}, \alpha\right\} & \text { if }\left(1, \bar{\alpha}_{1}\right)=\left\{\bar{\alpha}_{1}\right\} \\
\mathcal{A}\left(1, \alpha_{1}\right)= \begin{cases}\left\{\gamma_{1}, \beta_{1}, \alpha_{0}\right\} & \text { if } \alpha_{0} \neq \bar{\alpha}_{1}, \\
\left\{\beta_{1}, \alpha_{0}=\bar{\alpha}_{1}\right\} & \text { if } \alpha_{0}=\bar{\alpha}_{1} .\end{cases}
\end{array}
$$

CLAIM - One has

$$
\bigcup_{X} \mathcal{A}(0, \alpha) \supset X
$$

and equality holds only if $X=\left\{\alpha, \pi_{0}(\alpha)<k\right\}$ for some $k \leq \pi_{0}\left(\alpha_{1}\right)$ or $k=d$.

Similarly, one has, if $\alpha_{1} \notin X$

$$
\bigcup_{X} \mathcal{A}(1, \alpha) \supset X
$$

and equality holds only if $X=\left\{\alpha, \pi_{1}(\alpha)<k\right\}$, for some $k \leq d$.

The assertions of the claim are immediate from the definitions of $\mathcal{A}(\varepsilon, \alpha)$. We can now conclude the proof of the proposition. If $\hat{h}_{\alpha}=0$ for all $\alpha \in X$, we have $\operatorname{Im} \xi_{\beta}^{\varepsilon}=0$ for all $\beta \in \bigcup_{X} \mathcal{A}(\varepsilon, \alpha)$. When either $\bigcup_{X} \mathcal{A}(0, \alpha)$ or $\bigcup_{X} \mathcal{A}(1, \alpha)$ is strictly larger than $X$, we obtain the conclusion of the proposition. Otherwise, by the first half of the claim, we must have $X=\left\{\alpha, \pi_{0}(\alpha)<\right.$ $k\}$ for some $k \leq \pi_{0}\left(\alpha_{1}\right)$ or $k=d$. If $k \leq \pi_{0}\left(\alpha_{1}\right), \alpha_{1} \notin X$ and the second part of the claim would give $X=\left\{\alpha, \pi_{1}(\alpha)<k\right\}$, contradicting admissibility. 
Finally, in the remaining case $X=\mathcal{A}-\left\{\alpha_{0}\right\}$, one has $h_{\alpha}=0$ for all $\alpha \in \mathcal{A}$ (because $\hat{h}_{\alpha_{1}}=h_{\alpha_{1}}+h_{\alpha_{0}}$ ) and $\tau \equiv 0$.

\subsection{Checking integrability}

From the formula $(*)$ in section 5.4

$$
\begin{aligned}
& \mathcal{X}_{\pi_{0}, \pi_{1}}(\lambda)=\sum_{\varepsilon, \Gamma} \mathcal{X}_{\Gamma}(\lambda), \\
& \mathcal{X}_{\Gamma}(\lambda)=(d !)^{-1} \prod_{1}^{d}\left(\Sigma \hat{\lambda}_{\alpha} \hat{h}_{\alpha}^{(j)}\right)^{-1},
\end{aligned}
$$

we deduce the estimate, for each $\Gamma$ :

$$
c^{-1} \leq \mathcal{X}_{\Gamma}(\lambda) \prod_{j=1}^{d}\left(\sum_{W_{j}} \hat{\lambda}_{\alpha}\right) \leq c .
$$

When we restrict to $\left\{\hat{\lambda}^{*}=1\right\}$, the density up to a constant factor is given by the same formula. Let us decompose the simplex $\Delta:=\left\{\lambda, \hat{\lambda}_{\alpha}>0, \hat{\lambda}^{*}=1\right\}$ in the following way : the set of indices is

$$
\mathcal{N}=\left\{\mathbf{n}=\left(n_{\alpha}\right)_{\alpha \in \mathcal{A}} \in \mathbf{N}^{\mathcal{A}}, \min _{\alpha} n_{\alpha}=0\right\} .
$$

For each $\mathbf{n} \in \mathcal{N}$, denote by $\Delta(\mathbf{n})$ the set of $\left(\lambda_{\alpha}\right)_{\alpha \in \mathcal{A}} \in \Delta$ such that $\hat{\lambda}_{\alpha} \geq \frac{1}{2 d}$ if $n_{\alpha}=0$, and

$$
\frac{1}{2 d} 2^{1-n_{\alpha}}>\hat{\lambda}_{\alpha} \geq \frac{1}{2 d} 2^{-n_{\alpha}}
$$

if $n_{\alpha}>0$. We have a partition

$$
\Delta=\bigsqcup_{\mathcal{N}} \Delta(\mathbf{n}) .
$$

Clearly, we have, for $\mathbf{n} \in \mathcal{N}$

$$
c^{-1} \leq(\operatorname{vol} \Delta(\mathbf{n})) 2^{\Sigma n_{\alpha}} \leq c .
$$

On the other hand, for $\lambda \in \Delta(\mathbf{n})$ and $\Gamma$ as above, one obtains from (1) that

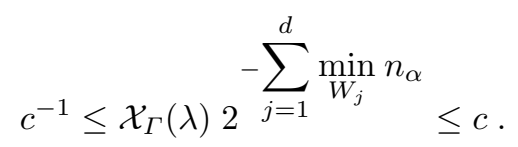

With fixed $\mathbf{n}$, let $0=n^{0}<n^{1}<\ldots$ be the values taken by the $n_{\alpha}$ and $V^{i} \subset \mathcal{A}$ the set of indices with $n_{\alpha} \geq n^{i}$. On one side, one has 


$$
\begin{aligned}
\sum_{\alpha} n_{\alpha} & =\sum_{i \geq 0} n^{i}\left(\#\left(V^{i}-V^{i+1}\right)\right) \\
& =\sum_{i>0}\left(n^{i}-n^{i-1}\right) \# V^{i} .
\end{aligned}
$$

On the other side, let $\tilde{V}^{i}$ be the set of $j$ such that $W_{j} \subset V^{i}$; one has $\min _{W_{j}} n_{\alpha}=n^{i}$ iff $j \in \tilde{V}^{i}-\tilde{V}^{i+1}$ hence

$$
\begin{aligned}
\sum_{j=1}^{d} \min _{W_{j}} n_{\alpha} & =\sum_{i \geq 0} n^{i}\left(\#\left(\tilde{V}^{i}-\tilde{V}^{i+1}\right)\right) \\
& =\sum_{i>0}\left(n^{i}-n^{i+1}\right) \# \tilde{V}^{i}
\end{aligned}
$$

By the Corollary of 6.5 , one has

$$
\# \tilde{V}^{i}<\# V^{i}
$$

as long as $0<\# V^{i}<d$. This shows that

$$
\sum_{\alpha} n_{\alpha}-\sum_{j=1}^{d} \min _{W_{j}} n_{\alpha} \geq|\mathbf{n}|_{\infty}:=\max _{\alpha} n_{\alpha} .
$$

The last estimate, introduced into (2), (3), gives

$$
(\operatorname{vol} \Delta(\mathbf{n})) \max _{\Delta(\mathbf{n})} \mathcal{X}_{\Gamma} \leq c 2^{-|\mathbf{n}|_{\infty}} .
$$

The integrability of $\mathcal{X}_{\Gamma}$ over $\Delta$ now follows from the fact that the number of $\mathbf{n} \in \mathcal{N}$ with $|\mathbf{n}|_{\infty}=N$ is of order $N^{d-2}$.

At the same time, we can see that the matrix $Z \in S L\left(\mathbf{Z}^{\mathcal{A}}\right)$ such that

$$
\lambda=Z \hat{\lambda}
$$

is such that $\log \|Z\|$ is integrable for the invariant measure $m$. We use as a norm the supremum of the coefficients. We have, for all $k \in \mathbf{N}$ (when $\varepsilon=0$; the case $\varepsilon=1$ is symmetric)

$$
\|Z\|>k \Longleftrightarrow \hat{\lambda}_{\alpha_{0}}>k \sum_{\pi_{1} \alpha>\pi_{1} \alpha_{0}} \hat{\lambda}_{\alpha},
$$

and therefore

$$
\|Z\|>(2 d) 2^{N-1} \Longrightarrow \lambda \in \bigcup_{|\mathbf{n}|_{\infty} \geq N} \Delta(\mathbf{n}) .
$$

This implies that

$$
\int_{\|Z\| \geq 2^{N}} \mathcal{X}_{\Gamma} \leq c N^{d-2} 2^{-N}
$$


for all $N>0$. Therefore $\|Z\|^{\rho}$ for $\rho<1$ and a fortiori $\log \|Z\|$ are integrable for the invariant measure $m$.

This integrability property puts us in position of applying Oseledets theorem and start studying the ergodic properties of the continuous fraction algorithm. However, we will restrain us to do that here.

\section{References}

1. J. Coffrey "Some remarks concerning an example of a minimal, non uniquely ergodic interval exchange map" Math. Z. 199 (1988) 577-580.

2. G. Forni "Solutions of the cohomological equation for area-preserving flows on compact surfaces of higher genus" Annals of Mathematics 146 (1997) 295-344.

3. G. Forni "Deviation of ergodic averages for area-preserving flows on surfaces of higher genus" Annals of Mathematics 155 (2002) 1-103.

4. A. Katok and A.M. Stepin "Approximations in Ergodic Theory" Russ. Math. Surv. 22 (1967) 77-102.

5. M. Keane "Interval exchange transformations" Math. Z. 141 (1975) 25-31.

6. M. Keane "Non-ergodic interval exchnage transformations" Isr. J. Math. 26 (1977) 188-196.

7. S.P. Kerckhoff "Simplicial systems for interval exchange maps and measured foliations" Ergod. Th. Dynam. Sys. 5 (1985) 257-271.

8. H.B. Keynes and D. Newton "A "Minimal", Non-Uniquely Ergodic Interval Exchange Transformation" Math. Z. 148 (1976) 101-105.

9. R. Krikorian "Déviations de moyennes ergodiques, d'après Forni, Kontsevich, Zorich" Séminaire Bourbaki 2003-2004, 56ème année, exposé $\mathrm{n}^{0} 927$, novembre 2003.

10. M. Kontsevich and A. Zorich "Connected components of the moduli spaces of Abelian differentials with prescribed singularities" Inv. Math. 153 (2003) 631-678.

11. H. Masur "Interval exchange transformations and measured foliations" Annals of Mathematics 115 (1982) 169-200.

12. S. Marmi, P. Moussa and J-C. Yoccoz "On the cohomological equation for interval exchange maps", C. R. Math. Acad. Sci. Paris 336 (2003) 941-948.

13. S. Marmi, P. Moussa and J-C. Yoccoz "The cohomological equation for Roth type interval exchange maps", preprint.

14. G. Rauzy "Echanges d'intervalles et transformations induites" Acta Arit. (1979) 315-328.

15. M. Rees "An alternative approach to the ergodic theory of measured foliations" Ergod. th. Dyn. Sys. 1 (1981) 461-488.

16. W. Veech "Interval exchange transformations" Journal d'Analyse Mathématique 33 (1978) 222-272.

17. W. Veech "Gauss measures for transformations on the space of interval exchange maps" Ann. of Math. 115 (1982) 201-242.

18. W. Veech "The Teichmuller geodesic flow" Ann. of Math. 124 (1986) 441-530.

19. W. Veech "The metric theory of interval exchange transformation I. Generic spectral properties" Amer. J. of Math. 106 (1984) 1331-1359 
20. W. Veech "The metric theory of interval exchange transformation II. Approximation by primitive interval exchanges " Amer. J. of Math. 106 (1984) 13611387

21. W. Veech "The metric theory of interval exchange transformation III. The Sah Arnoux Fathi invariant" Amer. J. of Math. 106 (1984) 1389-1421

22. A. Zorich "Finite Gauss measure on the space of interval exchange transformations. Lyapunov exponents" Annales de l'Institut Fourier Tome 46, fasc. 2 (1996) 325-370.

23. A. Zorich "Deviation for interval exchange transformations" Ergod. th. Dyn. Sys. 17 (1997), 1477-1499.

24. A. Zorich "On Hyperplane Sections of Periodic Surfaces" Amer. Math. Soc. Translations 179 (1997) 173-189.

25. A. Zorich "How Do the Leaves of a Closed 1-form Wind Around a Surface ?" in Pseudoperiodic Topology, V. Arnold, M. Kontsevich and A. Zorich editors, Amer. Math. Soc. Translations 197 (1999) 135-178. 